\title{
Integrated evaluation of telomerase activation and telomere maintenance across cancer cell lines
}

\author{
Kevin $\mathrm{Hu}^{1,2,3}$, Mahmoud Ghandi ${ }^{1+\ddagger}$, Franklin W Huang ${ }^{1,2,3 *}$ \\ ${ }^{1}$ Broad Institute of MIT and Harvard, Cambridge, United States; ${ }^{2}$ Division of \\ Hematology/Oncology, Department of Medicine; Bakar Computational Health \\ Sciences Institute; Institute for Human Genetics; University of California, San \\ Francisco, San Francisco, United States; ${ }^{3}$ Helen Diller Family Comprehensive Cancer \\ Center, San Francisco, United States
}

*For correspondence: franklin.huang@ucsf.edu

Present address: ${ }^{\dagger}$ Monte Rosa Therapeutics, Boston, United States; ${ }^{\ddagger}$ Cambridge Data Science LLC, Belmont, United States

Competing interest: See page 15

Funding: See page 15

Received: 02 January 2021 Preprinted: 24 January 2021 Accepted: 27 August 2021 Published: 06 September 2021

Reviewing editor: C Daniela Robles-Espinoza, International Laboratory for Human Genome Research, Mexico

(c) Copyright Hu et al. This article is distributed under the terms of the Creative Commons Attribution License, which permits unrestricted use and redistribution provided that the original author and source are credited.
Abstract In cancer, telomere maintenance is critical for the development of replicative immortality. Using genome sequences from the Cancer Cell Line Encyclopedia and Genomics of Drug Sensitivity in Cancer Project, we calculated telomere content across 1299 cancer cell lines. We find that telomerase reverse transcriptase (TERT) expression correlates with telomere content in lung, central nervous system, and leukemia cell lines. Using CRISPR/Cas9 screening data, we show that lower telomeric content is associated with dependency of CST telomere maintenance genes. Increased dependencies of shelterin members are associated with wild-type TP53 status. Investigating the epigenetic regulation of TERT, we find widespread allele-specific expression in promoter-wildtype contexts. TERT promoter-mutant cell lines exhibit hypomethylation at PRC2repressed regions, suggesting a cooperative global epigenetic state in the reactivation of telomerase. By incorporating telomere content with genomic features across comprehensively characterized cell lines, we provide further insights into the role of telomere regulation in cancer immortality.

\section{Introduction}

Telomeres, repetitive nucleoprotein complexes located at chromosomal ends, are an important component of genomic stability (de Lange, 2009). As protective chromosomal caps, telomeres prevent potentially lethal end-fusion events (McClintock, 1941; McClintock, 1942) and mis-processing of chromosomal ends as damaged sites by the DNA repair machinery (de Lange, 2005; Verdun and Karlseder, 2007). Due to factors such as incomplete DNA replication and oxidative stress, telomeres gradually shorten with successive rounds of cell division (Olovnikov, 1973). If left unchecked, telomere attrition eventually triggers growth arrest and senescence, and further shortening can lead to acute chromosomal breakage and cell death. Unrestricted telomere shortening therefore acts as a major obstacle in the course of tumor development (Hackett and Greider, 2002; Lorbeer and Hockemeyer, 2020), and inhibition of telomere maintenance offers still largely untapped opportunities for targeted cancer therapies (Damm et alı, 2001; Dikmen et al., 2005; Flynn et al., 2015).

Telomere shortening in embryonic development and in certain adult cell populations is offset by telomerase (Greider and Blackburn, 1985), a ribonucleoprotein enzyme with a core reverse transcriptase, TERT, that lengthens telomeres by catalyzing the addition of TTAGGG nucleotide repeats from an inbuilt RNA template component, TERC (Feng et al., 1995; Yu et al., 1990). Although telomerase is transcriptionally silenced in the majority of somatic cells, telomerase is reactivated in over $85 \%$ of all human cancers (Kim et al., 1994). Despite having activated telomere maintenance mechanisms, most cancers tend to have shorter telomeres than normal tissues, perhaps due to telomere 
maintenance mechanisms developing only after a critical state of telomere crisis has been reached (Okamoto and Seimiya, 2019). Reactivation of telomerase is associated with a diverse set of genomic alterations, the most common of which include highly recurrent mutations in the TERT promoter (Horn et al., 2013; Huang et al., 2013), aberrant methylation (Lee et al., 2019) or copy number amplification (Zhang et al., 2000) of TERT, and modulation of the numerous transcription factors that regulate TERT expression (Greider, 2012; Wu et alı, 1999). Of the minority of cancers that do not reactivate telomerase, many depend upon alternative lengthening of telomeres (ALT), a process that exploits mechanisms of homologous recombination and is characterized by heterogeneous telomere lengths, mutations in the ATRX and DAXX chromatin-regulating factors, and genome instability (Cesare and Reddel, 2010).

The readily identifiable nature of telomeric DNA repeats has motivated the development of computational methods for the determination of telomere content from whole-genome sequencing (WGS) and whole-exome sequencing (WES) data (UK10K Consortium et al., 2014). Recently, such methods were employed to characterize telomere content across tumor sequencing data from panels such as The Cancer Genome Atlas (TCGA), the Genotype-Tissue Expression (GTEx) project, and the Pan-Cancer Analysis of Whole Genomes (PCAWG) study, which have identified genomic markers of relative telomere lengthening and maintenance mechanisms (Barthel et al., 2017; Castel et al., 2019; PCAWG-Structural Variation Working Group et al., 2020). To gain a greater functional understanding of the landscape of telomere maintenance in cancer, we estimated telomeric DNA content (subsequently referred to as telomere content Feuerbach et al., 2019) across a diverse array of human cancer cell lines profiled in the Cancer Cell Line Encyclopedia (CCLE) (Barretina et al., 2012; Ghandi et al., 2019) and Genomics of Drug Sensitivity in Cancer (GDSC) (Yang et al., 2013) projects. Although cancer cell lines are immortalized, often through TERT activation, cell lines can serve as models for living tissues in aspects such as gene expression and have been deeply profiled (Barretina et al., 2012; Ghandi et al., 2019). We hypothesized that assessing telomere content could reflect underlying mechanisms of attrition, maintenance, and repair, which may be reflected in associations with genetic markers. By combining these estimates with a rich set of existing CCLE annotations, we aimed to determine genetic, epigenetic, and functional markers of telomere content and telomerase activity across a diverse panel of human cancer cell lines.

\section{Results}

\section{Telomere content across cancer cell lines}

Telomeric reads can be identified in DNA-sequencing reads using the canonical tandemly repeated TTAGGG motif, and normalized telomeric read counts may provide an accurate estimate of telomere content (UK10K Consortium et al., 2014). We quantified telomere content across cell lines using WGS and WES data from the independent CCLE (Barretina et al., 2012; Ghandi et al., 2019) and GDSC (Yang et al., 2013) datasets. In particular, we considered 329 cell lines profiled with WGS and 326 with WES in the CCLE, and 1056 samples profiled with WES in the GDSC, of which 55 were non-cancerous matched-normal samples. We note that our estimates state telomeric DNA repeat tract content, which is a normalized measure of telomeric reads in a sample, rather than solely telomere length, because telomere length requires the identification of true telomeric DNA from intrachromosomal, non-terminal telomeric DNA repeat tracts and extrachromosomal telomeric DNA (Feuerbach et al., 2019). To assess the fidelity of our telomere content measurements, we examined the agreement between the telomere content estimates in overlapping cell lines from independent sequencing datasets (Figure 1-figure supplements 1 and 2a). We observed high agreement between the telomere content estimates derived from CCLE WGS and GDSC WES data $(r=0.84, p$ $\left.=3.7 \times 10^{-79}, n=286\right)$ and moderate agreement between CCLE WGS and CCLE WES estimates ( $r=$ $0.71, p=1.5 \times 10^{-6}, n=36$ ), suggesting that our length estimates were not strongly biased by source. Among the three datasets used, CCLE WGS samples each captured at least ten thousand telomeric reads (which we estimated as those with six or more TTAGGG repeats), followed by GDSC WES samples at 100-1000 telomeric reads, and lastly by CCLE WES samples with 10-100 telomeric reads (Figure 1-figure supplement 2b). Moreover, one cell line (HEL 92.1.7) was twice-sequenced in the CCLE WGS dataset and these replicates had similar raw telomere length estimates (4.00 and 4.06 kilobases). Based on the agreement between CCLE WGS and GDSC WES, we generated a 
merged telomere content dataset of 1099 cell lines (Supplementary Methods) by combining the normalized log-transformed telomere contents derived from the CCLE WGS and GDSC WES datasets for downstream analyses.

Given these telomere content estimates, we first sought to examine the general distribution across cell lines and with respect to key cell line attributes such as donor age and population doubling rate. The overall distribution of telomere content displayed a slight skew (Figure 1-figure supplement 2a) toward longer telomeres, perhaps reflective of cell lines dependent upon ALT, a hallmark of which is telomeres of abnormal and heterogeneous lengths (Bryan et al., 1997; Heaphy et al., 2011b). We matched a substantial number of cell lines (282 for CCLE WGS, 554 for GDSC WES) with the age of the donor at the time of removal, from which we observed weak negative (vs. CCLE WGS: $r=-0.05, p=0.39$; vs. GDSC WES: $r=-0.17, p=6.0 \times 10^{-5}$ ) correlations between telomere content and the age of the original donor (Figure 1-figure supplement 3a). Similarly, we also found TC to be negatively correlated with the log CCLE-calculated cell line doubling rate (vs. CCLE WGS: $r=-0.17, p=0.01$; vs. GDSC WES: $r=-0.11, p=0.03$ ) (Figure 1-figure supplement $3 \mathrm{~b}$ ), consistent with shorter telomeres in cell lines modestly associated with higher doubling rates. Among 1099 merged CCLE WGS and GDSC WES samples, we found raw telomere content to vary substantially both between $\left(p=2.0 \times 10^{-15}\right.$, Kruskal-Wallis $H$ test) and within (Figure 1) cell lines of different primary sites (Figure 1-figure supplement $3 c, d$ ). Cell lines of hematopoietic origin (namely leukemias and lymphomas, which comprised 156 lines) tended to have higher telomere contents on average $\left(p=2.0 \times 10^{-8}\right.$, two-sided Mann-Whitney $U$ test), perhaps due to their elevated levels of telomerase expression, which were the highest among all subtypes. The 55 noncancerous samples profiled as part of the GDSC displayed relatively high telomere contents $(p=$ $5.9 \times 10^{-4}$, two-sided Mann-Whitney $U$ test, Figure 1), consistent with previous reports of widespread telomere shortening in cancer (Barthel et al., 2017). The greatest median telomere content, however, was found across lymphocyte and blood cell lines (Figure 1). The cell line with the highest telomere content was the U2-OS osteosarcoma line, a well-characterized model for ALT (Bryan et al., 1997).

\section{Genomic alterations associated with telomere content}

Having determined telomere content in the context of cell line meta-attributes, we next sought to leverage the substantial genomic profiles of the CCLE to find correlates of telomere content. To characterize the genomic signatures of telomere content, we correlated the CCLE WGS and GDSC WES telomere content estimates against molecular annotations in the CCLE, with a focus on alterations known to be associated with telomere maintenance. First, we observed that telomere content and TERT overall and isoform-specific mRNA levels were positively though weakly correlated as determined by RNA sequencing (RNA-seq) both within each subtype (Figure 1-figure supplement $4 a, b)$ and overall (Figure 1-figure supplement $4 c, d$ ). In specific cancer types such as CNS, lung, and leukemia, we found a higher correlation between TERT mRNA expression and telomere content (Figure 1-figure supplement $4 a, b$ ). Furthermore, we found negative associations between TERT mRNA levels and telomere content in bone and peripheral nervous system cell lines (Figure 1-figure supplement $4 a, b$ ). Because ALT is most commonly found in these cancer types, this may be a consequence of the near-mutual exclusivity between TERT expression and markers of ALT (Killela et al., 2013; Lee et al., 2018). Although mutations in ATRX and DAXX are closely associated with the development of the ALT phenotype (Brosnan-Cashman et al., 2018; Clynes et al., 2015; Heaphy et al., 2011a; ALT Starr Cancer Consortium et al., 2012; Ramamoorthy and Smith, 2015), comparisons between telomere content and mutations in ATRX and DAXX yielded significant associations only between DAXX alterations and merged telomere content (Figure 1-figure supplement 5a). We further repeated association tests with TP53, VHL, and IDH1 as identified previously among TCGA samples (Barthel et al., 2017) and confirm that truncating VHL mutations are associated with reduced telomere lengths (Figure 1-figure supplement 5a), although this may be confounded by the high occurrence of VHL mutations in kidney cell lines. Testing telomere content association with molecular features profiled in the CCLE, we identified several genes known to be associated with telomere biology, suggesting that our integration of telomere content with existing annotations could identify features relevant to telomere maintenance mechanisms (Figure 1-figure supplement $5 \mathrm{~b}$ ). While we found relatively few significant associations between telomere content and mutations, we note that we were limited to an absolute estimate of telomere content as 


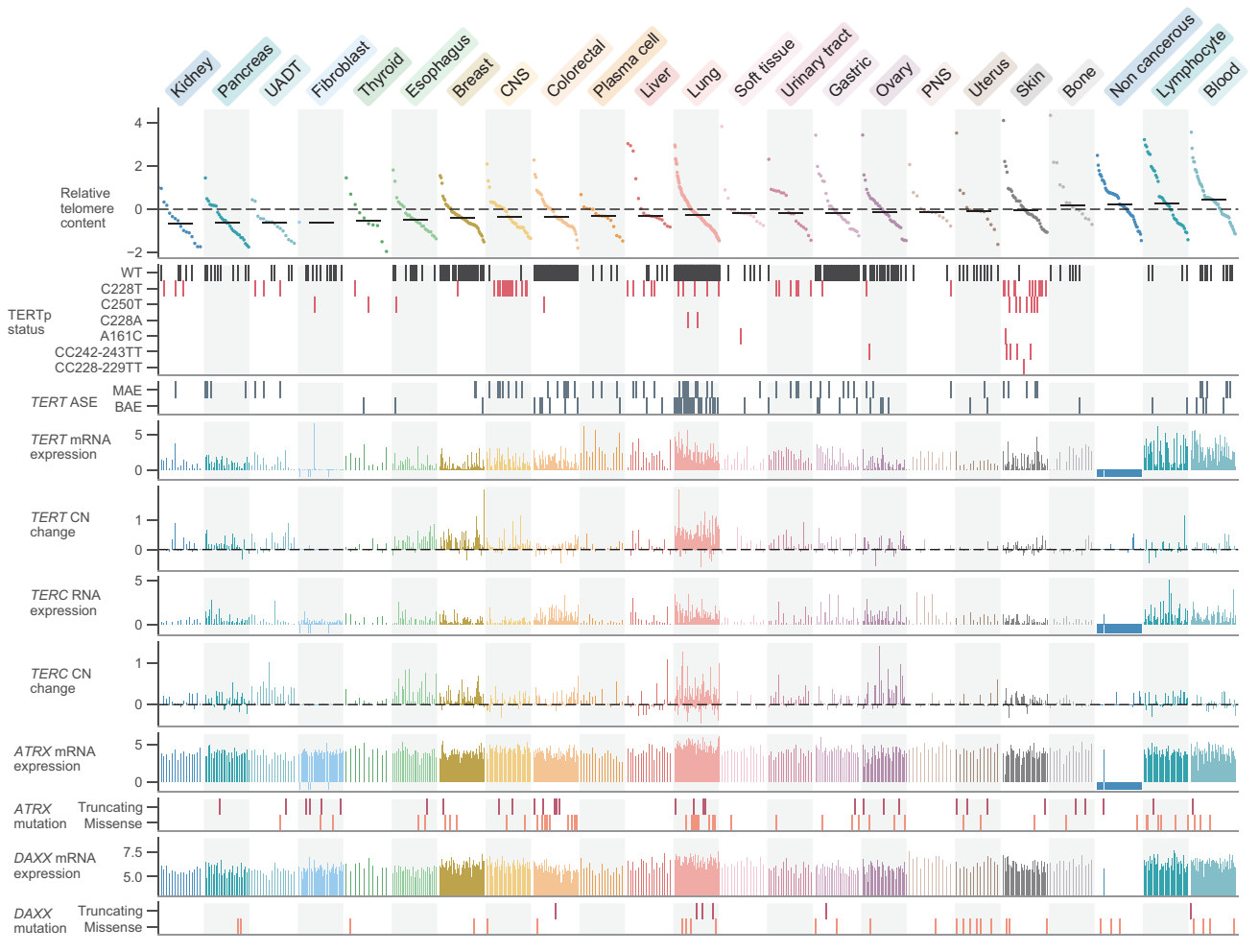

Figure 1. Telomere content and related genomic features across human cell lines. Cell lines were grouped by cancer type and ordered by telomere content within each type, and are displayed such that each column represents a cell line. Telomere content measurements reflect combined $z$-scored estimates derived from CCLE WGS and GDSC WES with means for samples with telomere content estimates from both sources. Bars within each cancer type represent medians. Relative copy number values are shown as $\log _{2}$ (relative to ploidy +1 ) -1 . Cell lines shown are filtered such in addition to annotations for telomere content, values for TERT and TERC RNA-seq expression, TERT and TERC copy number, and ATRX and DAXX mutation status are all available (with an exception made for non-cancerous cell lines, which lack such profiling in DepMap). Cell lines were also filtered such that each cancer type is represented by at least 10 cell lines ( $n=738$ cell lines total). RNA expression estimates are in terms of log 2 (TPM+1). CNS: central nervous system; PNS, peripheral nervous system; UADT, upper aerodigestive tract.

The online version of this article includes the following figure supplement(s) for figure 1:

Figure supplement 1. Overlap between cell lines represented in annotations.

Figure supplement 2. Telomere content agreement between sequencing sets.

Figure supplement 3. Telomere content, age, and tissue subtype.

Figure supplement 4. Transcriptomic associations between TERT, TERC, and telomere content.

Figure supplement $\mathbf{5}$. Associations between telomere content and cell line characteristics.

opposed to a relative measure of somatic telomere lengthening, which requires a paired normal sample (Barthel et al., 2017).

\section{Telomere content associates with CST complex dependencies}

Given the associations between telomere content and several genomic and transcriptomic features, we next considered whether variations in telomere content could confer or reduce selective vulnerabilities to inactivation of certain genes. In particular, we hypothesized that telomere content may be associated with vulnerabilities to reductions in the levels of telomere-regulating proteins. These vulnerabilities may be measured using CRISPR- and RNAi-based depletion assays, which can be processed to yield a numerical value for the dependency of a gene within a cell line, with more negative values indicative of increased dependence on a particular gene. To reveal such associations, we correlated our telomere content estimates with gene inactivation sensitivities assessed via genomewide CRISPR-Cas9 (Avana; Meyers et al., 2017) and RNAi viability screens (Achilles RNAi 
[Tsherniak et al., 2017] and DRIVE [McDonald et al., 2017]). Although we found no dependencies that displayed outlier associations with telomere content in the Achilles RNAi screen (Figure 1-figure supplement $\mathbf{5 b}$ ), we discovered that sensitivity to Avana CRISPR-Cas9 knockouts of each of the three CST complex proteins as well as the telomere-associating protein TERF1 were outlier associations with telomere content estimates computed from both the GDSC WES and CCLE WGS data (Figure 2a,b). Specifically, sensitivity to knockout of the CST complex components (CTC1, STN1, TEN1), which are key mediators of telomere capping and elongation termination (Chen et alo, 2012), was correlated with lower telomere content (Figure 2-figure supplement 1a,b). Although the CST complex was not assessed in the DRIVE screening dataset, we again found TERF1, a key shelterin component, to be among the positively correlated genes with telomere content in the

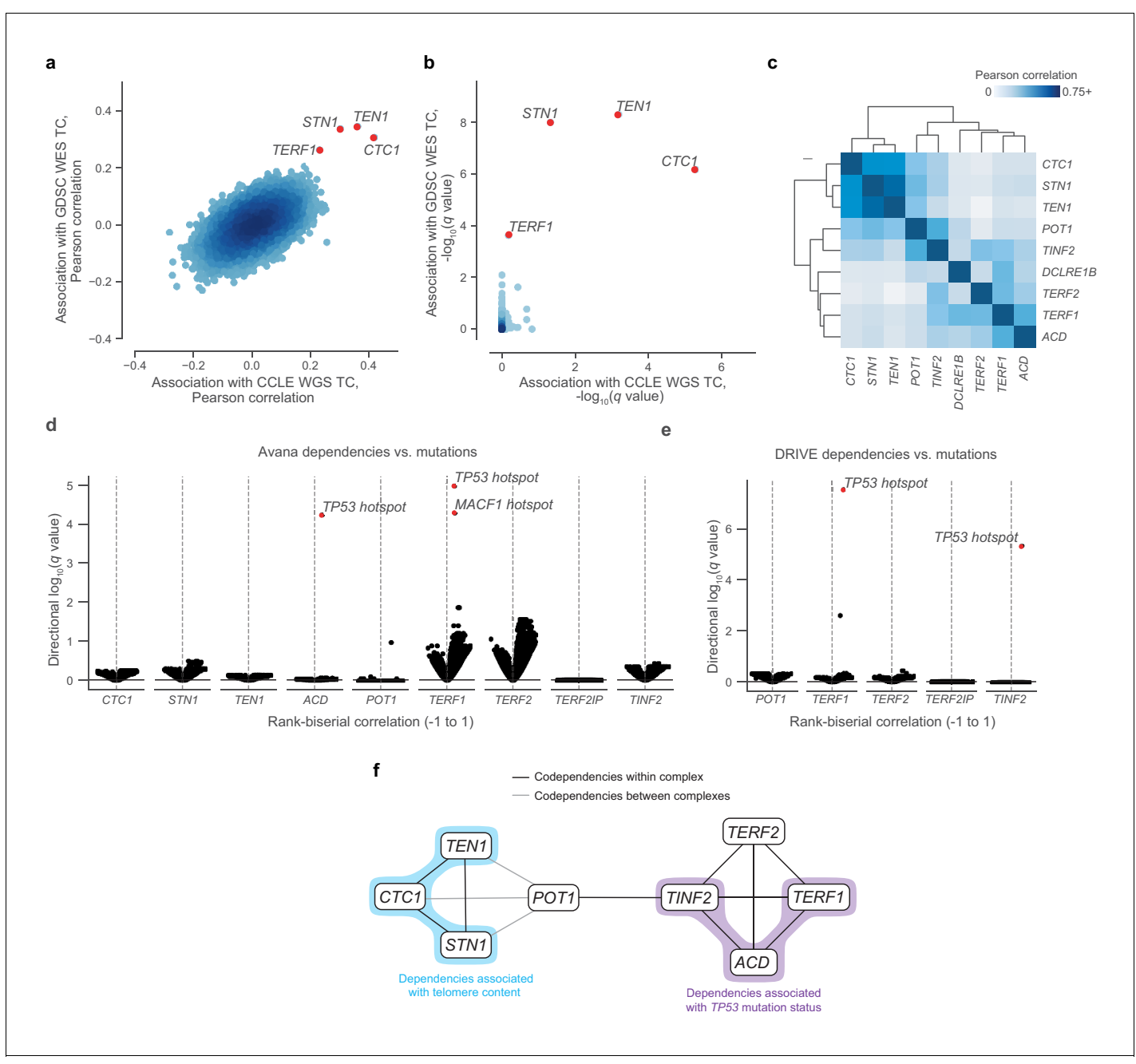

Figure 2. Telomere-binding protein dependencies are associated with telomere content and TP53 mutation status. (a) Pairwise plot of Pearson correlations between dependencies of all genes in the Avana dataset and CCLE WGS telomere content ( $x$-axis, $n=210-211$ cell lines) and GDSC WES telomere content ( $y$-axis, $n=420-426$ cell lines) estimates. (b) Pairwise plot of significance levels of correlations shown in (a) with correction for multiple hypothesis testing. (c) Pairwise Pearson correlation matrix between Avana dependencies among CST members and five shelterin components ( $n=796-$ 808 cell lines; Supplementary file 3). (d) Associations of CST and shelterin member Avana dependency scores with damaging and hotspot mutations ( $n$ $=796-808$ cell lines). For each gene dependency, mutation associations were computed using rank-biserial correlations with mutants and wild-types as the two categories. $p$ Values determined using two-sided Mann-Whitney $U$ test. (e) Associations of shelterin member DRIVE dependency scores with damaging and hotspot mutations ( $n=372-375$ cell lines; Supplementary file 3 ) under the same scheme used in (d). ( $f$ ) Network schematic of the codependency matrix shown in (c) and annotated with association with telomere content or TP53 mutation status.

The online version of this article includes the following figure supplement(s) for figure 2 :

Figure supplement 1. Telomere content and telomere protein dependencies.

Figure supplement 2. TP53 mutation status and shelterin member dependencies. 
DRIVE panel (Supplementary file 2). Overall, these data suggest that cancer cell lines with shorter telomeres are more susceptible to inhibition of the CST-mediated telomere maintenance mechanism.

Using the CST complex as a seed set, we subsequently queried all dependencies under the premise that associated gene dependencies reflect coordinated functions (Pan et al., 2018). Within the Avana panel ( $n=757-769$ ), we found significant (FDR $<0.01$ ) outlier associations between the CST complex genes and genes encoding six additional telomere-associating proteins (ACD, POT1, TERF1, TERF2, TINF2, and DCLRE1B). These first five additional telomere-associating proteins, together with TERF2IP, comprise the shelterin complex, the protector and regulator of telomere length and topology (de Lange, 2005). Interestingly, whereas the five other shelterin dependencies were positively associated with telomere content, TERF2IP displayed a weak negative association (Figure 2-figure supplement 1c), suggesting that TERF2IP may play a distinct regulatory role in shelterin function compared to the other members. To examine the dependency landscape of the CST complex and these six other telomere-related proteins, we computed a correlation matrix involving these nine genes, clustering of which yielded two main subgroups: one comprised of the CST complex members, and another of the six other genes (Figure 2c). Despite this separation, POT1 and TINF2 also displayed notable correlations with CST dependencies, possibly serving as the primary mediators of previously-reported functional interactions between the shelterin and CST complexes (Chen et al., 2012; Wan et al., 2009).

Although we found strong codependency relationships within this group of telomere-associated proteins, we also observed that certain shelterin members displayed notable codependencies with p53 pathway members such as MDM2, ATM, and TP53 itself (Figure 2-figure supplement 2a,b). Because sensitivity to perturbation of the p53 pathway is highly associated with TP53 mutations in cancer (McDonald et al., 2017), we asked if these codependency relationships were also associated with hotspot mutations in TP53. In fact, TP53 was a significant (FDR $<0.001)$ outlier when a comprehensive set of hotspot and damaging mutations was compared against sensitivity to ACD and TERF1 dependencies in the Avana panel (Figure 2d, Figure 2-figure supplement 2c) and against TERF1 and TINF2 dependencies in the DRIVE panel (Figure 2e, Figure 2-figure supplement 2d). These links between these gene dependencies and TP53 mutation status reprise and extend previous reports of p53-dependent DNA damage responses to TERF1 and TINF2 depletion (Pereboeva et al., 2016; Rosenfeld et al., 2009). Taken together, we find that CST and shelterin dependencies are correlated with each other, telomere content, and TP53 mutation status (Figure 2f).

\section{Patterns and mechanisms of telomerase expression}

Having thoroughly characterized telomere content and its related dependencies across the CCLE, we next focused on the regulation of TERT transcription itself. Across 1019 samples previously profiled with deep RNAseq, we found that hematopoietic cell lines (leukemias, lymphomas, and myelomas) were associated with the greatest mean expression of TERT ( $p=5.8 \times 10^{-26}$, two-sided MannWhitney $U$ test; Figure 1). In contrast, TERT expression was significantly reduced $\left(p=2.0 \times 10^{-22}\right.$, two-sided Mann-Whitney $U$ test) and generally undetectable in fibroblast-like cell lines. With regard to the telomerase RNA component (TERC), we found strong associations between TERC RNA expression and RNA levels of several small Cajal body-specific RNAs (scaRNAs) and histone subunit RNAs (Figure 1-figure supplement 4e). The co-expression of TERC and these other RNAs may be a consequence of their shared localization, processing, and regulation in Cajal bodies (Gall, 2003; Venteicher et al., 2009; Zhu et al., 2004), as TERC itself contains an H/ACA box small nucleolar RNA (snoRNA) domain (Mitchell et al., 1999) and is a scaRNA family member. Moreover, these associations suggest that variations in Cajal body processing may act as factors in TERC reactivation (Cao et al., 2008). Although we focused on the transcriptional features of telomerase, it is important to note that other factors in addition to TERT and TERC expression determine telomerase activity and the eventual maintenance of telomere length (Listerman et al., 2013). Despite these orthogonal factors, TERT enzymatic activity remains strongly correlated with raw levels of TERT expression (Takakura et al., 1998).

Beyond raw gene and transcript expression levels, we next examined the underlying mechanisms for reactivating TERT in cancer using the comprehensive cell line data. Widespread transcriptional reactivation of TERT in cancer is driven by a variety of factors. Aside from copy number 
amplifications (Zhang et al., 2000), highly recurrent mutations in the TERT promoter drive strong monoallelic TERT re-expression (Bell et alı, 2015; Huang et alı, 2015). To explore the intersections between methylation, promoter mutations, and allele-specific expression, we combined available profiling data from the CCLE (Figure 3a). First, using WGS and targeted sequencing of the TERT promoter provided in the CCLE, we assessed TERT promoter status for 503 cell lines across 21 cancer types (Figure 3-figure supplement 1a). We found that only the C228T (chr5:1,295,228 C>T) mutation was significantly ( $p=2.8 \times 10^{-5}$, two-sided Mann-Whitney $U$ test) associated with an increase in TERT expression (Figure 3-figure supplement $1 \mathrm{~b}$ ). Surprisingly, the mean level of TERT expression in monoallelic contexts was only slightly lower than that of biallelic contexts, with less
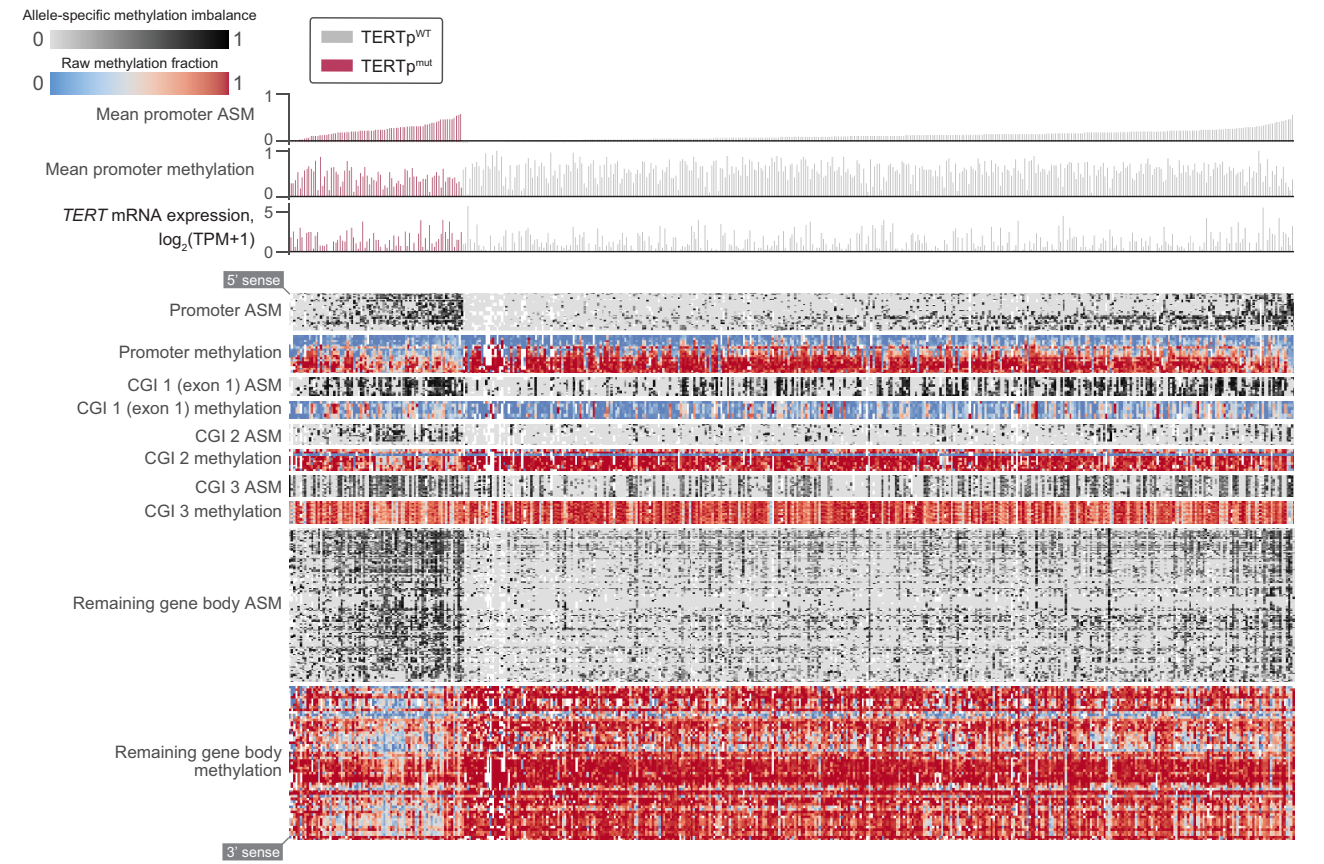

b

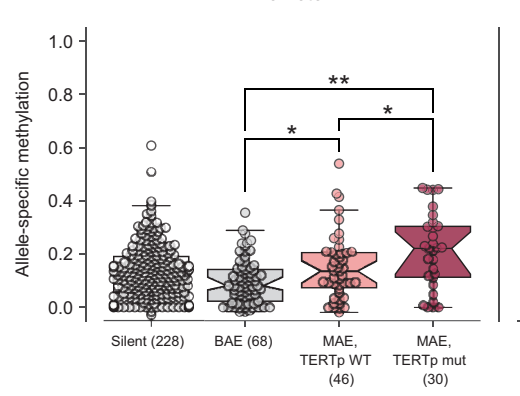

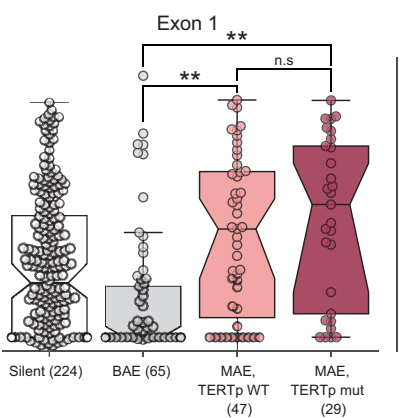

Remaining gene body

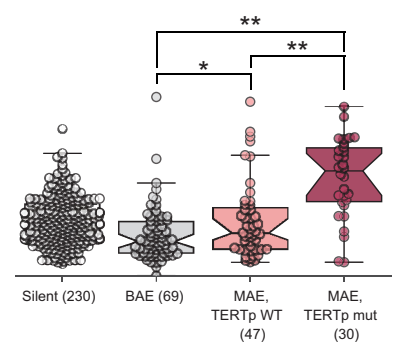

Figure 3. Allele-specific methylation of the TERT locus is indicative of both promoter mutation status and allele-specific expression. (a) Heatmap of CPG methylation levels along the TERT locus, sorted in order of mean methylation levels along the upstream $5 \mathrm{~kb}$ region within TERTp-mutants and wildtypes. TERT gene expression levels are also indicated for each cell line. Each column represents a cell line $(n=450)$, and each row represents a CpG pair $(n=209)$ sorted from the $5^{\prime}$ to $3^{\prime}$ direction along the TERT sense strand. White blocks indicate missing ASM/methylation values. Cell lines with unavailable ASM values for at least half of TERT locus CpGs were excluded. (b) ASM levels of TERT locus subregions in cell lines are indicative of TERTp status and allele-specific expression. BAE, biallelic expression; MAE, monoallelic expression. Boxes, interquartile range (IQR); center lines, median; whiskers, maximum and minimum or $1.5 \times$ IQR; notches, 95\% confidence interval of bootstrapped median using 1000 samples and a Gaussian-based asymptotic approximation. ${ }^{*} \mathrm{p}<0.05,{ }^{\star *} \mathrm{p}<0.01, \mathrm{n} . \mathrm{s}$, not significant; two-sided Mann-Whitney $U$ test.

The online version of this article includes the following figure supplement(s) for figure 3 :

Figure supplement 1. TERT promoter mutations, gene expression, and ASE.

Figure supplement 2. Interactions bewteen TERT ASE, promoter mutations, and methylation. 
than a 1.5 -fold difference between the groups ( $p=0.03$, two-sided Mann-Whitney $U$ test). Given that cells with biallelic TERT expression presumably express TERT with twice the transcriptional source sites as those with monoallelic TERT expression, this reduced difference may be a consequence of the effects of the TERT promoter mutation in producing particularly robust monoallelic expression (Huang et al., 2013) or expression of TERT from multiple sites all of the same allele (Rowland et al., 2019).

To further explore allele-specific expression (ASE) patterns of TERT, we employed an ASE-calling pipeline (Supplementary Methods) and determined TERT allele-specific expression status for 157 cell lines (Figure 3-figure supplement 1c), an increase of 69 cell lines compared to a previous report using CCLE WGS data (Huang et al., 2015). Out of these 157 cell lines, 87 (58.6\%) express TERT from a single allele. Moreover, of these 157 cell lines, 129 have a sequenced promoter, with which we confirm that promoter mutations unanimously drive monoallelic expression (Figure 3-figure supplement 2a). Our expanded set of cell lines also reveals several new tissues of origin in which TERT is monoallelically expressed without a mutant promoter, such as hematopoietic cell lines (Figure 3-figure supplement 2b). This high proportion of TERT monoallelic expression led us to ask whether there are genomic alterations aside from promoter mutations that could lead to ASE. Under the assumption that such alterations may also induce ASE in a larger region than a promoter mutation, we determined ASE status in SLC6A19 and CLPTM1L, which are the most immediate neighbor genes of TERT. Because the number of samples with annotated ASE in both TERT and these neighbors was not large enough for comparisons of overlapping ASE, we instead examined the individual frequencies of ASE among these three genes. Compared to promoter-wildtype monoallelic TERT expression occurring in $36 \%$ ( 47 of 129) of samples, only $9.4 \%$ (11 out of 117) of samples expressed CLPTM1L from a single allele and 22\% (5 of 22) expressed SLC6A19 from a single allele, and we were unable to assess co-occurrence due to lack of overlap in samples with heterozygous SNPs in both TERT and CLPTM1L or SLC6A19. Furthermore, a search for structural variants in the surrounding 100 kilobase regions yielded no significant associations. However, given that only 106 cell lines had both a known TERT ASE status and the required WGS data for structural variant determination, more genomic annotations may be needed for the discovery of additional mechanisms driving the monoallelic expression of TERT.

\section{Distinct TERTp methylation patterns at the TERT locus}

Aside from monoallelic expression, TERT promoter mutations are characterized by unique patterns of epigenetic marks, namely allele-specific CpG methylation (ASM) and H3K27me3 repressive histone modifications (Stern et al., 2015; Stern et al., 2017) and long-range chromatin interactions (Akıncılar et alo, 2016). Using genome-wide RRBS data across 928 cell lines, we elucidated associations between CpG-site methylation of the TERT locus (namely, TERT and the surrounding $5 \mathrm{~kb}$ ) and TERT promoter mutations. Examination of methylation patterns at the TERT locus revealed five prominent ASM clusters in the TERT locus, corresponding roughly to the upstream $5 \mathrm{~kb}$ region (containing the promoter), part of a CpG island overlapping the first exon, two other parts of this CpG island, and the remaining gene body (Figure 3a). Comparison of each region's mean ASM against TERTp mutant status revealed that TERTp mutants exhibited strong and significant $(p<0.01)$ increases in ASM in the promoter $(n=485)$, remaining gene body $(n=493)$, and exon $1(n=478)$ regions (Figure 3b, Figure 3-figure supplement 2c). In contrast, TERTp wild-type cell lines tended to lack ASM throughout the TERT locus, instead being hypermethylated in all regions except for exon 1 (Figure 3b, Figure 3-figure supplement 2d), and partial hypomethylation in promoter mutants may reflect the hemizygous methylation previously observed at the TERT locus (Rowland et al., 2020; Stern et al., 2015; Stern et al., 2017). Absolute methylation of the remaining gene body was positively correlated with TERT expression in both promoter status contexts (Figure 3-figure supplement 1e), which parallels previous reports of a positive correlation between TERT expression and methylation (Barthel et al., 2017; Salgado et al., 2019). Exon 1 methylation was elevated in nearly all cell lines with monoallelic TERT expression in both the mutant promoter context $\left(p=6.3 \times 10^{-3}\right.$, two-sided Mann-Whitney $U$ test, $\left.n=81\right)$ and the wildtype promoter context $\left(p=1.6 \times 10^{-4}\right.$, two-sided Mann-Whitney $U$ test, $\left.n=98\right)$ compared to biallelic TERT expressors (Stern et alo, 2017). Interestingly, although most cell lines with monoallelic TERT expression displayed partially elevated methylation levels in exon 1 (Figure 3b), only promoter-mutant cell lines 
were hypomethylated in the surrounding regions, suggesting that the epigenetic state of promoter mutants is in fact distinct from that of promoter-wildtype monoallelic TERT expressors.

\section{A genome-wide epigenetic pattern in TERTp mutants}

The observation that TERT promoter mutants display a hypomethylated TERT locus even compared to other monoallelic TERT expressors led us to ask if additional epigenetic signals are indicative of TERT promoter status. In particular, we considered the possibility that epigenetic changes to the TERT locus could in fact act as a cooperative factor (Kim et al., 2016; Kim and Shay, 2018) in tumorigenesis or tumor cell maintenance rather than as a consequence of TERT promoter mutations. To address this hypothesis, we performed a genome-wide search for CpG islands (CGls) with significant differences in methylation levels in TERTp mutant cell lines compared to TERTp wild-type ones. If TERT hypomethylation were a downstream consequence of TERT promoter mutations then we would expect TERT hypomethylation to be an isolated event, and thus there would be few CGls outside the vicinity of TERT with methylation levels correlated with TERTp mutant status. Surprisingly, we instead found a broad genome-wide distribution of CGls that were hypomethylated in TERTp ${ }^{\text {mut }}$

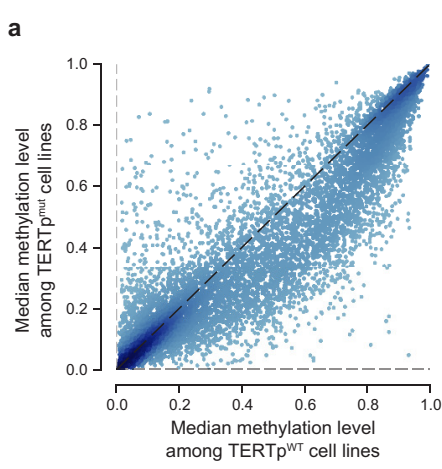

e

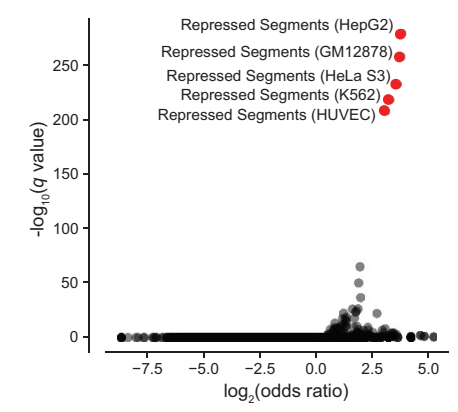

b
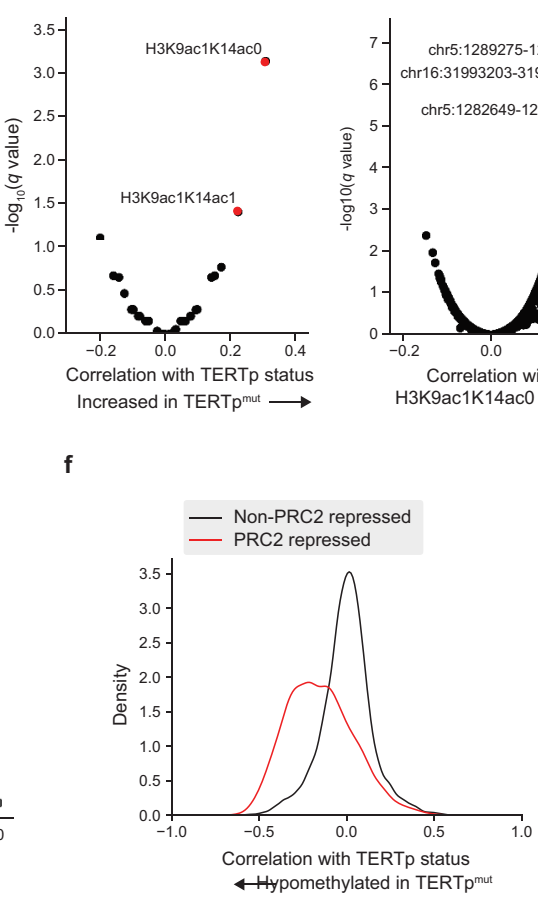
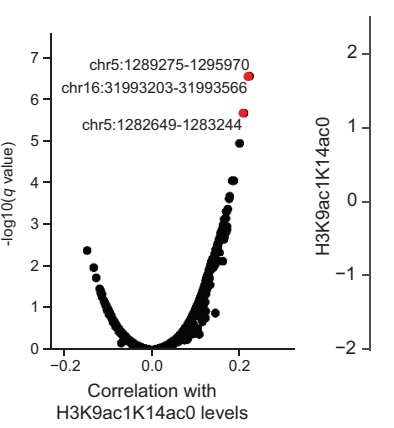

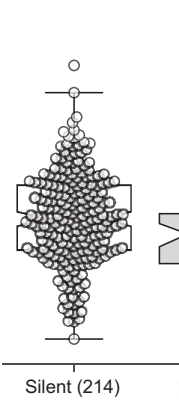

Silent (214)

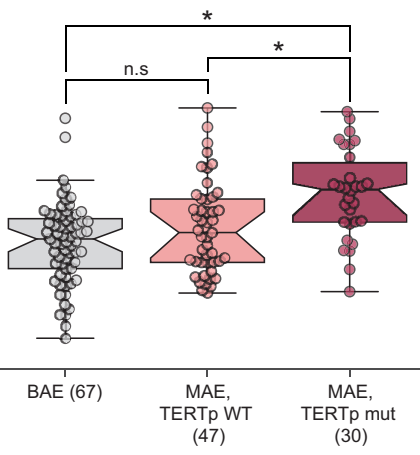

g

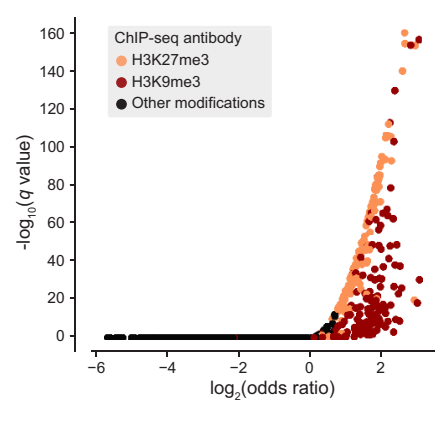

Figure 4. TERT promoter mutations associate with genome-wide decreased methylation of PRC2-repressed regions. (a) Pairwise plot of median CGI methylation levels in TERTp ${ }^{\text {mut }}$ cell lines ( $n=21-83$; Supplementary file 6) versus TERTp ${ }^{\text {WT }}$ cell lines $(n=95-410$, Supplementary file 6). Each dot represents a CGI. (b) Rank-biserial correlations between TERTp status (mutant or wild-type) and global histone modification levels ( $n=302-475)$. Significance determined by two-sided Mann-Whitney $U$ test. (c) Pearson correlation levels between global H3K9ac1K14ac0 levels and ASM imbalance of CGIs $(n=261-884)$. (d) H3K9ac1K14ac0 levels are significantly increased in TERTp mutants. Boxes, interquartile range (IQR); center lines, median; whiskers, maximum and minimum or $1.5 \times$ IQR; notches, 95\% confidence interval of bootstrapped median using 1000 samples and a Gaussian-based asymptotic approximation. ${ }^{*} p<0.01$, n.s, not significant; two-sided Mann-Whitney $U$ test. (e) LOLA core set enrichment analysis of CGls hypomethylated in TERTP ${ }^{\text {mut }}$ cell lines reveals enrichment of PRC2-repressed regions. (f) Kernel density distributions of rank-biserial correlations between CGI methylation levels for PRC2-overlapping regions and non-PRC2-overlapping regions. A negative correlation indicates that a CGI is hypomethylated in TERTp ${ }^{\text {mut }}$ cell lines relative to TERTp ${ }^{\text {WT }}$ ones, and a positive correlation indicates the opposite. PRC2 regions were sourced from the HepG2 segmentation. (g) LOLA ENCODE Roadmap region enrichment analysis of CGls hypomethylated in TERTp ${ }^{\text {mut }}$ cell lines reveals enrichment of H3K9me3 and H3K27me3 regions.

The online version of this article includes the following figure supplement(s) for figure 4:

Figure supplement 1. Global methylation changes associated with TERT promoter mutations. 
samples relative to TERTp WT samples (Figure 4a). Moreover, when correlated with a panel of global histone modification levels, we found that TERTp mutants exhibited increased levels of $\mathrm{H} 3 \mathrm{~K} 9 \mathrm{ac} 1 \mathrm{~K} 14 \mathrm{ac0}$ and H3K9ac1K14ac1 marks (Figure 4b), which have been suggested as marks of transcriptionally active chromatin (Ruthenburg et al., 2007). Likewise, when H3K9ac1K14ac0 levels were compared against a genome-wide panel of CGI ASM levels, the TERT CGI (chr5:1,289,275$1,295,970$ ) was the top correlate (Figure 4c). H3K9ac1K14ac0 levels were significantly increased in TERTp mutants compared to monoallelic TERTp wild-type cell lines, linking this histone modification to TERTp mutation (Figure $4 d$ ).

To better understand the distribution of these TERTp ${ }^{\text {mut }}$-hypomethylated CGIs, we utilized Locus Overlap Analysis (LOLA) (Nagraj et al., 2018; Sheffield and Bock, 2016) to query the significance of overlaps between these CGls and predetermined region sets. Among the top 1000 TERTp ${ }^{\text {mut }}$ hypomethylated CGIs (Figure 4a), we found significant (FDR $<0.0001$ ) and robust 10 -fold enrichment for polycomb repressive complex 2 (PRC2)-repressed regions (Figure 4e) previously characterized in several cell lines (HepG2, GM12878, HeLa-S3, K562, and HUVEC). Beyond these top 1000 hypomethylated CGls, CGls overlapping with PRC2-repressed segments were broadly hypomethylated in TERTp ${ }^{\text {mut }}$ cell lines and accounted for nearly all the previously observed skew toward hypomethylation (Figure 4f). Interestingly, the enrichment of PRC2 segments was much smaller (around 3.5-fold) in the remaining profiled cell line, H1-hESC. Against ENCODE ChIP-seq peak region sets, we also found significant overlap with the H3K9me3 and H3K27me3 heterochromatin marks (Figure 4g). Furthermore, we also observed a moderate twofold ( $P=5.1 \times 10^{-24}$, Fisher's exact test; Figure 4-figure supplement 1a) enrichment for regions within 10 megabases of most telomeres, consistent with previous reports that PRC2-repressed and H3K27me3-marked regions are enriched in telomeric and subtelomeric regions (Rosenfeld et al., 2009). The enrichment of these hypomethylated regions among telomere-proximal regions may also be indicative of a recently-reported telomere position effect, which has been shown to affect the chromatin accessibility of the TERT locus (Kim et al., 2016) located close to the chromosome $5 \mathrm{p}$ telomere. Given that PRC2 has previously been shown to exhibit allele-specific binding to the methylated silent allele in TERTp ${ }^{\text {mut }}$ cell lines (Stern et al., 2017), this genome-wide pattern of hypomethylation at PRC2 sites suggests that background epigenetic events may interact with promoter mutations in facilitating TERT expression.

Given this peculiar pattern of hypomethylation at telomere-proximal sites of PRC2 repression across CCLE samples, we next asked if a similar pattern exists across TCGA primary tumor samples. To test this hypothesis, we estimated CGI methylation levels across 878 TERT-expressing TCGA samples characterized with both the Illumina $450 \mathrm{k}$ array and with a previously determined TERT promoter status (Barthel et al., 2017). Consistent with previous analyses of TERT methylation levels at the cg11625005 methylation probe, we find that TERTp ${ }^{\text {mut }}$ samples tended to exhibit hypomethylation (Figure 4-figure supplement 1b,c). Among 13,547 CGls, we again found an enrichment of hypomethylation of PRC2-overlapping CGls (Figure 4-figure supplement 1d), although this was less prominent than previously noted in the CCLE. LOLA enrichment analysis for TERTp ${ }^{\text {mut }}$-hypomethylated CGIs in the TCGA likewise confirmed significant enrichments (FDR < 0.0001) of PRC2repressed regions and associated histone modifications as the top enriched region sets (Figure 4figure supplement 1e,f). However, the fold-enrichment was less (about five-fold) than that observed in the CCLE and did not display any significant enrichment in telomere-proximal regions $(p=0.70$, Fisher's exact test). Although the skew toward hypomethylation in TERT promoter mutants among these TCGA samples was weaker than in the CCLE samples, this may be the result of the more heterogeneous nature of primary TCGA samples as well as the differences in coverage between the Illumina $450 \mathrm{k}$ array and RRBS.

\section{Discussion}

To investigate the nature of telomeres and their maintenance mechanisms in cancer, we applied a functional genomics approach toward understanding molecular relationships across cancer cell lines. We estimated relative telomere content across over a thousand cancer cell lines and thus provide a useful reference for further studies on cancer cell line characteristics that have not to date considered this feature. We show that cell line telomere content indeed varies with factors such as tissue type, TERT mRNA expression, and mutations in genes such as DAXX and VHL. Moreover, we discovered novel relationships between telomere content and dependencies of CST and shelterin complex 
members, which was enabled by the high overlap between the cell lines profiled by our estimates and by several loss-of-function screens (McDonald et al., 2017; Meyers et al., 2017; Tsherniak et al., 2017).

Using these genome-wide gene dependency estimates, we found that increased sensitivity (Meyers et al., 2017) to depletion of CST complex members correlates with shorter telomeres, likely a consequence of the critical roles of the CST complex in both telomere protection and in terminating telomere elongation (Chen et al., 2012). Our findings raise the possibility that targeting the CST complex may preferentially affect cancer cells that harbor shorter telomeres, and telomere content may be used as a biomarker of drug response in tumors. Likewise, CST complex dependencies were positively associated with the dependencies of several shelterin complex components, reflecting their functional interactions. Among these shelterin complex members, we find that the responses to their depletion are highly dependent upon the presence of a wild-type TP53 gene, with TP53 mutants displaying reduced sensitivity to depletion of ACD, TERF1, and TINF2. Additional studies are required to validate these associations and to assess why only certain members of the shelterin complex show this TP53-dependent sensitivity effect.

In addition to telomere content, we also investigated the genomic landscape of telomere maintenance mechanisms, namely mechanisms of TERT reactivation, across cancer cell lines. The enrichment of TERT promoter mutations in certain tissues has inspired several explanations, and our findings in both the CCLE and TCGA suggest a specific epigenetic signature that may underlie this unique pathway of telomere maintenance. We found that in TERT promoter mutants, CpG islands were preferentially hypomethylated in PRC2-repressed regions located near telomeres, which may relate to previous reports of a long-range telomere position effect (Kim et al., 2016; Yuan et al., 2019) and of TERT expression necessitating specific chromatin states in promoter-wildtype and mutant samples (Salgado et alı, 2019). Considering that normal tissues typically exhibit particularly low methylation of the TERT promoter (Salgado et alı, 2019; Stern et alı, 2017) and that PRC2 occupies the inactive allele in TERT promoter mutants (Stern et al., 2017), our genome-wide signature may relate to the latter part of the two-step mechanism proposed for TERTp mutation-driven telomerase upregulation (Chiba et al., 2017). Moreover, epigenetic mechanisms have been shown to produce synergistic effects with driver mutations in tumor evolution (Tao et al., 2019). Besides reflecting a direct cooperation with TERT expression, this signature raises the possibility that the 'memory' of short telomeres may be preserved through these telomere-proximal hypomethylated regions. It may also be indicative of the stemness of cell lines, which has been proposed as a major factor in the proliferative advantage of TERT promoter mutations (Chiba et alı, 2015). Future studies will be necessary to elucidate the nature of this epigenomic signature, how it impacts the regulation of telomerase expression, and the complexities of TERT expression beyond binary measures of allele-specificity (Rowland et al., 2019). Furthermore, incorporation of telomere content into studies using cancer cell lines may help improve our understanding of sensitivities to drugs or genetic perturbations across cell lines.

Through our analysis, we show relevant markers of telomere-associated protein function, patterns of TERT reactivation across cancers, and epigenetic determinants of TERT promoter status. We detail various features of telomere regulation and dysfunction in cancer, and we provide a substantial addition of new features to a well-characterized set of cell lines. By doing so, we complement molecular studies of telomeres in parallel studies across the GTEx (GTEx Consortium et al., 2020), TCGA (Barthel et al., 2017), and PCAWG (PCAWG-Structural Variation Working Group et al., 2020) panels, providing a resource that will guide additional studies on the roles and functions of telomeres in cancer.

\section{Materials and methods}

\section{Telomere content estimation}

Telomere content estimates were computed using Telseq (UK10K Consortium et al., 2014) with the default settings. Telseq records the frequencies of reads containing various frequencies of the canonical TTAGGG telomeric repeat, and then normalizes this number of telomeric repeats using a GC-adjusted coverage estimate and the average chromosome length. 
Telomere content was estimated for WGS and WES samples in the CCLE (Ghandi et al., 2019) as well as WES samples in the GSDC (Yang et al., 2013) using the default settings. When multiple read groups were present in a sample, telomere content was computed as a mean of the individual read group estimates weighted by the total read count per group. Whereas we found decent agreement between overlapping samples in CCLE WGS and GDSC WES, we found a comparatively weak correlation between both sets and the CCLE WES estimates (Figure 1-figure supplement 2). Therefore, we excluded the CCLE WES telomere content estimates from subsequent analyses.

In comparing the CCLE WGS and GDSC WES estimates, we also noticed a batch effect resulting in two clusters of GDSC WES estimates. To identify and correct this batch effect across all GDSC WES estimates, we observed that these batches were distinguished by frequencies of reads containing exactly 4, 5, and 6 telomeric motifs. We then ran a k-means clustering on these read frequencies to estimate the clusters across all GDSC WES samples, which were subsequently adjusted by re-centering the mean of one cluster (after applying a z-scored log-transformation) to match the mean of the other.

We also attempted to use Telseq to estimate telomeric repeat-containing RNA (TERRA) expression across 1019 RNA-seq samples from the CCLE. However, because the majority of these samples were found to contain little or no reads containing telomeric reads, TERRA capture was determined to be too low for any meaningful analysis.

Cell lines were annotated with sample descriptors from the CCLE data portal (Cell_lines_annotations_20181226.txt, https://portals.broadinstitute.org/ccle/data). Harmonized sample information, telomere content estimates, and other matched annotations are available in Supplementary file 1.

\section{Genomic and transcriptomic markers}

We sourced mutations and copy number estimates from the DepMap download portal (https://depmap.org/portal/download/) under the public 1904 release (CCLE_mutations.csv and CCLE_gene_cn.csv, respectively). We used the mutation classifications detailed in the Variant_annotation column.

We also downloaded processed RNAseq estimates in the form of gene expression, transcript expression, and exon inclusion estimates from the CCLE data portal under the latest release (CCLE_RNAseq_rsem_genes_tpm_20180929.txt.gz,

CCLE_RNAseq_rsem_transcripts_tpm_20180929.txt.gz, and CCLE_RNAseq_ExonUsageRatio_20180929.gct.gz, respectively). We also downloaded RPPA estimates (CCLE_RPPA_20181003.csv) and global chromatin profiling results (CCLE_GlobalChromatinProfiling_20181130.csv). Before performing subsequent analyses, we transformed transcript and gene expression TPMs by taking a $\log _{2}$-transform with a pseudocount of +1 . We also excluded transcripts with a standard deviation in this $\log _{2}(T P M+1)$ measure of less than 0.25 across all cell lines. We excluded exons with missing inclusion values in over 800 cell lines or with a standard deviation of less than 0.1. Pearson correlations were then used to calculate associations between gene and transcript RNA expression levels of TERT and TERC against telomere content estimates as well as other markers. We also constructed linear models regressing merged telomere content as a function of various biomarkers with cell line primary site as a covariate. Moreover, we also calculated linear models of each of CCLE and GDSC telomere content as a function of TERT mRNA levels, TERC mRNA levels, primary site, TERTp mutant status, donor age, and calculated doubling time to examine the contributions of these specific factors. Out of these factors, we found TERT and TERC mRNA levels to be significantly correlated with both CCLE and GDSC telomere contents.

We also considered processed methylation estimates available on the CCLE data portal, namely the TSS $1 \mathrm{~kb}$ upstream estimates as well as the promoter CpG cluster estimates, which we correlated against telomere content estimates. For these annotations, we filtered out regions with a standard deviation of less than 0.05 .

Results of TERT and TERC expression associations, as well as telomere content associations, are available in Supplementary file 2.

\section{Gene dependency associations}

To identify gene dependencies associated with telomere content, we considered knockout/knockdown effects estimated in the Avana CRISPR-Cas9 (Meyers et al., 2017), Achilles RNAi 
(Tsherniak et al., 2017), and DRIVE RNAi (McDonald et al., 2017) datasets. For Achilles and DRIVE, the datasets used were the April 2020 versions listed on the DepMap portal computed with DEMETER2. For Avana, we used gene effect scores from the February 2021 release. For each gene dependency in each dataset, we computed the Pearson correlation coefficient against telomere content estimates generated separately with CCLE WGS and GDSC WES data. Correlation $p$ values were determined using the two-tailed Student's $t$ test. All correlation coefficients and $p$ values were determined using the pearsonr function as part of the scipy.stats Python module.

To identify codependencies with the CST complex members, we employed an iterative approach to identify highly ranked correlations. In particular, starting with a seed set of genes (the base case of which was the CST complex), we searched for codependencies between two genes $x$ and $y$ under the criteria that the $r^{2}$ association between the two is among the top five for $x$ vs. all other genes, and among the top five for $y$ vs. all other genes as well. We recursively applied this method four times, which added the five shelterin components ACD, POT1, TERF1, TERF2, and TINF2 to our gene set. To construct the clustered correlation matrix in Figure 2c, we used the clustermap function as provided by the Seaborn Python library, with Ward's method for the determination of the hierarchical clustering.

To identify significant associations between dependencies and mutations, we compared dependencies against binary categories of damaging/truncating (comprised of deleterious alterations, such as nonsense and splice-site alterations) and hotspot (highly recurrent) mutations. Using the previously downloaded DepMap 1904 mutation annotations, we considered mutations as 'damaging'/'truncating' if they were associated with a 'damaging' label under the Variant_annotation column, and we considered mutations as 'hotspot' if they were labeled as such in the corresponding COSMIC (Tate et al., 2019) (isCOSMIChotspot) or TCGA (isTCGAhotspot) columns. We excluded mutations with a total damaging or hotspot frequency of less than five across all profiled CCLE samples. Mutations were then compared with dependencies using a two-sided Mann-Whitney $U$ test, with the two classes being non-damaging and non-hotspot mutant samples, and damaging and hotspot mutant samples, respectively.

To rank and visualize the codependencies shown in Figure 2-figure supplement $2 a, b$ and the dependency-mutation associations shown in Figure $2 d$,e, we used a signed $q$-value approach. We first transformed the raw false discovery rates by taking the negative of the base-10 logarithm, and we then applied a sign to this transformed value as determined by the direction of the codependency (the sign of the correlation coefficient) or dependency-mutation association (negative for greater sensitivity in mutants, and positive otherwise).

Dependency analyses results are available in Supplementary file 3.

\section{Characterization of allele-specific TERT expression}

Allele-specific expression may be detected by looking for discordant counts of reads mapping to single-nucleotide polymorphisms (SNPs) in DNA-sequencing vs. RNA-sequencing reads (Huang et al., 2015). In particular, allele-specific expression is evidenced by the biased frequency of a single allele of a heterozygous SNP in RNAseq reads compared to that of DNA-sequencing reads. To assess TERT expression in the context of allele-specificity, we examined cell lines for which DNA (WES or WGS) and RNA (RNAseq) sequencing data were available. To identify heterozygous anchor SNPs, we considered mutations in the TERT gene body called using Mutect 1.1.6 (Cibulskis et al., 2013) with default settings. We then applied a filter for mutations with at least eight reads supporting both the reference and alternate alleles that passed the Mutect quality control filter (i.e. classified as PASS). To force call the matching allele frequencies in RNA, we processed the matching aligned RNAseq reads using the ASEReadCounter tool provided in GATK 3.6 (Van der Auwera et al., 2013) with arguments -minDepth 8, -minBaseQuality 16, -minMappingQuality 255, and -U ALLOW_N_CIGAR_READS.

We then used these RNA and DNA allele frequencies to classify cell lines as monoallelic and biallelic expressors of TERT as well as two neighboring genes, SLC6A19 and CLPTM1L. In particular, we examined the odds ratio derived from a binary contingency table with the two sets of categories being the context (DNA vs. RNA) and the allele (reference vs. alternate) of the read counts. To account for edge cases where the denominator of the odds ratio was zero, we added a pseudocount of 0.5 to each category before computing the odds ratio. We then denoted MAE lines as those having an odds ratio computed using the major allele as the denominator of greater than five. In 
instances where there were multiple informative SNPs, we considered only the SNP with the greatest supporting total RNA-seq read count. In cases where the same SNP was detected across multiple sources (for instance, in both CCLE WES and WGS), we considered the source with the greatest coverage of the SNP.

Allele-specific calls for TERT, SLC6A19, and CLPTM1L are described in Supplementary file 4.

\section{Genome-wide allele-specific methylation analysis}

To characterize and compare CpG-level ASM around the TERT genomic region, we utilized RRBS data generated by the CCLE (Ghandi et al., 2019). Mapped BAM files were downloaded from the CCLE FireCloud workspace, and ASM levels for each CpG pair were estimated using the allelicmeth command from the MethPipe package (Song et alı, 2013). Within each sample, we first included only CpG pairs with a minimum coverage of eight reads. Next, among all 928 cell lines, CpG pairs included in less than $5 \%$ of these samples were excluded.

To estimate ASM, we employed a strategy similar to the original MethPipe ASM pipeline. For each pair of $\mathrm{CpGs}$, we considered the four combinations of methylation states between the two CpGs: methylated-methylated $(\mathrm{mm})$, methylated-unmethylated $(\mathrm{mu})$, unmethylated-unmethylated (uu), and unmethylated-unmethylated ( $\mathrm{mm}$ ). For semi-methylated $\mathrm{CpG}$ pairs not subject to ASM, we would expect high and relatively equal frequencies of the mu and um pairs, whereas for ASM CpG pairs, we would expect the allele bias to result in high $\mathrm{mm}$ and uu counts and low um and $\mathrm{mu}$ counts. To quantify this imbalance, we used the mean square contingency coefficient $(\Phi)$ with a pseudocount of 0.5. Namely, for each CpG pair, we computed

$$
\Phi=\frac{m m * u u-m u * u m}{\sqrt{(\dot{m m}+\dot{m} u)(\dot{u} m+\dot{u} u)(m m+\dot{m})(\dot{m u}+\dot{u} u)}}
$$

where $m m=m m+0.5, m u=m u+0.5, u m=u m+0.5$, and $u u=u u+0.5$. ASM CpG pairs therefore had a positive $\Phi$, whereas non-ASM pairs had a $\Phi$ of around 0 . We rounded negative $\Phi$ values to 0 . Before computing these imbalance values, we excluded $\mathrm{CpG}$ pairs with a methylation level of less than 0.1 or greater than 0.9 on either CpG, so as to filter out CpG pairs that were likely to be fully methylated or completely demethylated.

We first examined the ASM levels of the TERT locus, which considered as the TERT gene body as well as the flanking five kilobase regions. For these methylation estimates, we excluded $\mathrm{CpGs}$ with less than 25\% valid ASM estimates. We segmented these CpGs into five regions: the promoter (chr5:1295246-1298643), CGI_1 (chr5:1294872-1295134), CGI_2 (chr5:1291374-1294439), CGI_3 (chr5: 1289695-1291090), and the remaining gene body (chr5: 1249661-1289359). We computed ASM imbalance values for these regions by taking the mean CpG-pair ASM values.

To identify genome-wide methylation events indicative of TERT promoter mutations, we searched for correlates with TERT promoter mutation status among average methylation levels of $\mathrm{CpG}$ islands (CGIs). CpG island annotations were downloaded from the UCSC genome browser at http:// hgdownload.cse.ucsc.edu/goldenpath/hg19/database/cpglslandExt.txt.gz. To filter out low-coverage $\mathrm{CpG}$ islands, we considered only $\mathrm{CpG}$ islands with at least eight $\mathrm{CpG}$ sites. Methylation levels per island were then estimated by taking the mean across all $\mathrm{CpGs}$ profiled within the island. Using the same filtering parameters, we also computed mean ASM estimates across these CGls.

TERT locus methylation estimates are described in Supplementary file 5.

\section{Chromatin profiling data}

To identify histone modifications associated with TERTp status, we downloaded global chromatin profiling data from the CCLE Data Portal (CCLE_GlobalChromatinProfiling_20181130.csv). Correlations between TERTp status and histone modification levels, as well as correlations between H3K9ac1K14ac0 levels and CGI ASM levels, are described in Supplementary file 5.

\section{Region enrichment analysis}

To characterize the regions that were hypomethylated in association with TERT promoter mutations, we utilized Locus Overlap Analysis (LOLA) (Nagraj et al., 2018; Sheffield and Bock, 2016), which 
discovers enriched regions among a background set. LOLA takes as input two region sets: the regions of interest and a background universe set. Both sets are then overlapped against a database of annotated regions, and overlapping and non-overlapping region frequencies are computed per annotated region set. Region overlap significance is then assessed against each annotated region set using Fisher's exact test with the two categories being the regions of interest vs. the background universe set and the overlap of each of these regions with the annotated region set.

We ranked hypomethylated CpG island regions by the significance of the change in TERTp wild type vs. TERTp mutant cell lines as assessed by a two-sided Mann-Whitney $U$ test (i.e. regions with the most significant changes were ranked first). The top $1000 \mathrm{CpG}$ islands were then used as the regions of interest, and the set of all $\mathrm{CpG}$ islands examined served as the background universe set. We utilized the LOLAweb application (at http://lolaweb.databio.org/), with the LOLACore and LOLARoadMap sets as the region databases.

Outputs of LOLA on CCLE TERT promoter-mutant hypomethylated CGls are summarized in Supplementary file 6.

\section{TCGA data}

TCGA methylation and normalized gene expression estimates were downloaded from the UCSC Xena browser (Goldman et al., 2019) (http://xena.ucsc.edu/, jhu-usc.edu_PANCAN_HumanMethylation450.betaValue_whitelisted.tsv.synapse_download_5096262.xena and EB++AdjustPANCAN_IlluuminaHiSeq_RNASeqV2.geneExp.xena). Methylation levels of CGls was estimated by averaging CpG methylation levels within each CGI, and CGls with less than four profiled CpGs were excluded. TERT promoter mutation status was obtained from previous estimates (Barthel et al., 2017). The same hypomethylation-enrichment analysis previously described for the CCLE was then run on the top $1000 \mathrm{CGls}$ hypomethylated in TERT promoter-mutants using an identical ranking scheme.

A summary of LOLA results on TCGA methylation, TERT promoter status, and ALT-likelihood is provided in Supplementary file 7.

\section{Statistical analysis}

Multiple hypothesis testing was accounted for using the Benjamini-Hochberg FDR with an alpha of 0.01 as provided by the statsmodels Python module.

\section{Acknowledgements}

We thank Dr. Elizabeth Blackburn (UCSF) and Dr. Thomas Cech (University of Colorado Boulder) for providing insightful comments and feedback on the manuscript. We thank Dr. Hani Goodarzi (UCSF) for his generosity in providing storage and computing resources. We thank members of the Huang lab for their feedback.

\section{Additional information}

Competing interests

Mahmoud Ghandi: MG is an employee and holds equity in Monte Rosa Therapeutics and is a founding member at Cambridge Data Science LLC. The other authors declare that no competing interests exist.

Funding

\begin{tabular}{lll} 
Funder & Grant reference number & Author \\
\hline Prostate Cancer Foundation & Young Investigator Award & Franklin W Huang \\
\hline
\end{tabular}

The funders had no role in study design, data collection and interpretation, or the decision to submit the work for publication. 
Author contributions

Kevin Hu, Data curation, Software, Formal analysis, Investigation, Visualization, Methodology, Writing - original draft, Writing - review and editing; Mahmoud Ghandi, Conceptualization, Resources, Data curation, Software, Formal analysis, Supervision, Investigation, Visualization, Methodology, Project administration, Writing - review and editing; Franklin W Huang, Conceptualization, Resources, Supervision, Funding acquisition, Investigation, Methodology, Writing - original draft, Project administration, Writing - review and editing

Author ORCIDs

Kevin Hu (10) https://orcid.org/0000-0002-3631-8294

Mahmoud Ghandi (iD https://orcid.org/0000-0003-1897-2265

Franklin W Huang (D) https://orcid.org/0000-0001-5447-0436

Decision letter and Author response

Decision letter https://doi.org/10.7554/eLife.66198.sa1

Author response https://doi.org/10.7554/eLife.66198.sa2

\section{Additional files}

Supplementary files

- Supplementary file 1. Telomere content estimates and sample information.

- Supplementary file 2. Telomere content and transcriptomic associations.

- Supplementary file 3. Unsupervised dependency associations.

- Supplementary file 4. Allele-specific expression calls.

- Supplementary file 5. TERT locus methylation.

- Supplementary file 6. Genome-wide methylation analysis in the CCLE.

- Supplementary file 7. Genome-wide methylation analysis in TCGA.

- Transparent reporting form

Data availability

Telomere content estimates can be found in the supplementary materials and have been uploaded to the Cancer Dependency Map portal (https://depmap.org/portal/).

The following previously published datasets were used:

\begin{tabular}{|c|c|c|c|c|}
\hline Author(s) & Year & Dataset title & Dataset URL & $\begin{array}{l}\text { Database and } \\
\text { Identifier }\end{array}$ \\
\hline $\begin{array}{l}\text { Ghandi M, Huang } \\
\text { FW, Jané-Valbuena } \\
\text { J, Kryukov GV, Lo } \\
\text { CC, McDonald ER, } \\
\text { Barretina J, Gelfand } \\
\text { ET, Bielski CM, Li } \\
\text { H, Hu K, Andreev- } \\
\text { Drakhlin AY, Kim J, } \\
\text { Hess JM, Haas BJ, } \\
\text { Aguet F, Weir BA, } \\
\text { Rothberg MV, } \\
\text { Paolella BR, } \\
\text { Lawrence MS, } \\
\text { Akbani R, Lu Y, Tiv } \\
\text { HL, Gokhale PC, } \\
\text { Weck S, } \\
\text { MansourAA, Oh C, } \\
\text { Shih J, Hadi K, } \\
\text { Rosen Y, Bistline J, } \\
\text { Venkatesan K, } \\
\text { Reddy A, Sonkin D, } \\
\text { Liu M, Lehar J, } \\
\text { Korn JM, Porter }\end{array}$ & 2019 & Cancer Cell Line Encyclopedia & $\begin{array}{l}\text { https://portals.broadin- } \\
\text { stitute.org/ccle }\end{array}$ & Broad Institute, ccle \\
\hline
\end{tabular}


DA, Jones MD,

Golji J, Caponigro

G, Taylor JE,

Dunning CM,

Creech AL, Warren

AC, McFarland JM

Zamanighomi M,

Kauffmann A,

Stransky N,

Imielinski $\mathrm{M}$,

Maruvka YE,

Cherniack $A D$

Tsherniak Z,

Vazquez $F$, Jaffe

JD, Lane AA

Weinstock DM,

Johannessen CM,

Morrissey MP.

Stegmeier $F$,

Schlegel R, Hahn

WC, Getz G, Mills

GB, Boehm JS,

Golub TR,

Garraway LA,

Sellers WR

Nusinow DP, Szpyt
J, Ghandi M, Rose
CM, McDonald ER

2020 Quantitative Proteomics of the Cancer Cell Line Encyclopedia

Kalocsay M, Jané-

Valbuena J,

Gelfand E,

Schweppe DK

Jedrychowski M,

Golji J, Porter DA

Rejtar T, Wang YK,

Kryukov GV

Stegmeier $F$

Erickson BK

Garraway LA

Sellers WR, Gygi SP

\begin{tabular}{|c|c|c|c|c|}
\hline DepMap & 2020 & DepMap 2002 Public & $\begin{array}{l}\text { https://depmap.org/por- } \\
\text { tal/download/ }\end{array}$ & $\begin{array}{l}\text { Dep Map Portal, } \\
\text { depmap.org/portal/ } \\
\text { download/ }\end{array}$ \\
\hline $\begin{array}{l}\text { lorio F, Knijnenburg } \\
\text { TA, Vis D, Bignell } \\
\text { G, Menden M, } \\
\text { Schubert M, Aben } \\
\text { N, Gonçalves E, } \\
\text { Barthorpe S, } \\
\text { Lightfoot H, } \\
\text { Cokelaer T, } \\
\text { Greninger P, Chang } \\
\text { H, Silva H, Heyn H, } \\
\text { Deng X, Egan RK, } \\
\text { Liu Q, Mironenko T, } \\
\text { Mitropoulos X, } \\
\text { Richardson L, Wang } \\
\text { J, Zhang T, Moran } \\
\text { S, Sayols S, } \\
\text { Soleimani M, } \\
\text { Tamborero T, } \\
\text { López-Bigas N, } \\
\text { Ross-MacDonald P, } \\
\text { Esteller M, Gray N, } \\
\text { Haber D, Stratton } \\
\text { MR, Benes C, } \\
\text { Wessels L, Saez- } \\
\text { Rodriguez J, } \\
\text { McDermott U, } \\
\text { Garnett M }\end{array}$ & 2016 & $\begin{array}{l}\text { GDSC whole-exome sequencing } \\
\text { data }\end{array}$ & $\begin{array}{l}\text { https://www.ebi.ac.uk/ } \\
\text { ega/studies/EGA } \\
\text { S00001000978 }\end{array}$ & $\begin{array}{l}\text { European Genome- } \\
\text { phenome Archive, } \\
\text { EGAS00001000978 }\end{array}$ \\
\hline
\end{tabular}

https://gygi.hms.harvard. Gygi Lab, gygi.hms. edu/publications/ccle. harvard.edu/ html publications/ccle. html 


\section{References}

Akıncılar SC, Khattar E, Boon PL, Unal B, Fullwood MJ, Tergaonkar V. 2016. Long-Range chromatin interactions drive mutant TERT promoter activation. Cancer Discovery 6:1276-1291. DOI: https://doi.org/10.1158/21598290.CD-16-0177, PMID: 27650951

ALT Starr Cancer Consortium, Lovejoy CA, Li W, Reisenweber S, Thongthip S, Bruno J, de Lange T, De S, Petrini JH, Sung PA, Jasin M, Rosenbluh J, Zwang Y, Weir BA, Hatton C, Ivanova E, Macconaill L, Hanna M, Hahn WC, Lue NF, Reddel RR, et al. 2012. Loss of ATRX, genome instability, and an altered DNA damage response are hallmarks of the alternative lengthening of telomeres pathway. PLOS Genetics 8:e1002772. DOI: https://doi. org/10.1371/journal.pgen.1002772, PMID: 22829774

Barretina J, Caponigro G, Stransky N, Venkatesan K, Margolin AA, Kim S, Wilson CJ, Lehár J, Kryukov GV, Sonkin D, Reddy A, Liu M, Murray L, Berger MF, Monahan JE, Morais P, Meltzer J, Korejwa A, Jané-Valbuena J، Mapa FA, et al. 2012. The Cancer cell line encyclopedia enables predictive modelling of anticancer drug sensitivity. Nature 483:603-607. DOI: https://doi.org/10.1038/nature11003, PMID: 22460905

Barthel FP, Wei W, Tang M, Martinez-Ledesma E, Hu X, Amin SB, Akdemir KC, Seth S, Song X, Wang Q, Lichtenberg T, Hu J, Zhang J, Zheng S, Verhaak RG. 2017. Systematic analysis of telomere length and somatic alterations in 31 Cancer types. Nature Genetics 49:349-357. DOI: https://doi.org/10.1038/ng.3781, PMID: 2 8135248

Bell RJ, Rube HT, Kreig A, Mancini A, Fouse SD, Nagarajan RP, Choi S, Hong C, He D, Pekmezci M, Wiencke JK, Wrensch MR, Chang SM, Walsh KM, Myong S, Song JS, Costello JF. 2015. The transcription factor GABP selectively binds and activates the mutant TERT promoter in Cancer. Science 348:1036-1039. DOI: https://doi. org/10.1126/science.aab0015, PMID: 25977370

Brosnan-Cashman JA, Yuan M, Graham MK, Rizzo AJ, Myers KM, Davis C, Zhang R, Esopi DM, Raabe EH, Eberhart CG, Heaphy CM, Meeker AK. 2018. ATRX loss induces multiple hallmarks of the alternative lengthening of telomeres (ALT) phenotype in human glioma cell lines in a cell line-specific manner. PLOS ONE 13:e0204159. DOI: https://doi.org/10.1371/journal.pone.0204159, PMID: 30226859

Bryan TM, Englezou A, Dalla-Pozza L, Dunham MA, Reddel RR. 1997. Evidence for an alternative mechanism for maintaining telomere length in human tumors and tumor-derived cell lines. Nature Medicine 3:1271-1274. DOI: https://doi.org/10.1038/nm1197-1271, PMID: 9359704

Cao Y, Bryan TM, Reddel RR. 2008. Increased copy number of the TERT and TERC telomerase subunit genes in Cancer cells. Cancer Science 99:1092-1099. DOI: https://doi.org/10.1111/j.1349-7006.2008.00815.x, PMID: 1 8482052

Castel SE, Aguet F, Mohammadi P, Consortium G, Ardlie KG, Lappalainen T. 2019. A vast resource of allelic expression data spanning human tissues. bioRxiv. DOI: https://doi.org/10.1101/792911

Cesare AJ, Reddel RR. 2010. Alternative lengthening of telomeres: models, mechanisms and implications. Nature Reviews Genetics 11:319-330. DOI: https://doi.org/10.1038/nrg2763, PMID: 20351727

Chen LY, Redon S, Lingner J. 2012. The human CST complex is a terminator of telomerase activity. Nature 488 : 540-544. DOI: https://doi.org/10.1038/nature11269, PMID: 22763445

Chiba K, Johnson JZ, Vogan JM, Wagner T, Boyle JM, Hockemeyer D. 2015. Cancer-associated TERT promoter mutations abrogate telomerase silencing. eLife 4:e07918. DOl: https://doi.org/10.7554/eLife.07918

Chiba K, Lorbeer FK, Shain AH, McSwiggen DT, Schruf E, Oh A, Ryu J, Darzacq X, Bastian BC, Hockemeyer D. 2017. Mutations in the promoter of the telomerase gene TERT contribute to tumorigenesis by a two-step mechanism. Science 357:1416-1420. DOI: https://doi.org/10.1126/science.aao0535, PMID: 28818973

Cibulskis K, Lawrence MS, Carter SL, Sivachenko A, Jaffe D, Sougnez C, Gabriel S, Meyerson M, Lander ES, Getz G. 2013. Sensitive detection of somatic point mutations in impure and heterogeneous Cancer samples. Nature Biotechnology 31:213-219. DOI: https://doi.org/10.1038/nbt.2514, PMID: 23396013

Clynes D, Jelinska C, Xella B, Ayyub H, Scott C, Mitson M, Taylor S, Higgs DR, Gibbons RJ. 2015. Suppression of the alternative lengthening of telomere pathway by the chromatin remodelling factor ATRX. Nature Communications 6:8538. DOI: https://doi.org/10.1038/ncomms8538, PMID: 26143912

Damm K, Hemmann U, Garin-Chesa P, Hauel N, Kauffmann I, Priepke H, Niestroj C, Daiber C, Enenkel B, Guilliard B, Lauritsch I, Müller E, Pascolo E, Sauter G, Pantic M, Martens UM, Wenz C, Lingner J, Kraut N, Rettig WJ, et al. 2001. A highly selective telomerase inhibitor limiting human Cancer cell proliferation. The EMBO Journal 20:6958-6968. DOI: https://doi.org/10.1093/emboj/20.24.6958, PMID: 11742973

de Lange T. 2005. Shelterin: the protein complex that shapes and safeguards human telomeres. Genes \& Development 19:2100-2110. DOI: https://doi.org/10.1101/gad.1346005, PMID: 16166375

de Lange T. 2009. How telomeres solve the end-protection problem. Science 326:948-952. DOI: https://doi.org/ 10.1126/science.1170633, PMID: 19965504

Dikmen ZG, Gellert GC, Jackson S, Gryaznov S, Tressler R, Dogan P, Wright WE, Shay JW. 2005. In vivo inhibition of lung Cancer by GRN163L: a novel human telomerase inhibitor. Cancer Research 65:7866-7873. DOI: https://doi.org/10.1158/0008-5472.CAN-05-1215, PMID: 16140956

Feng J, Funk WD, Wang SS, Weinrich SL, Avilion AA, Chiu CP, Adams RR, Chang E, Allsopp RC, Yu J. 1995. The RNA component of human telomerase. Science 269:1236-1241. DOI: https://doi.org/10.1126/science.7544491, PMID: 7544491

Feuerbach L, Sieverling L, Deeg KI, Ginsbach P, Hutter B, Buchhalter I, Northcott PA, Mughal SS, Chudasama P, Glimm H, Scholl C, Lichter P, Fröhling S, Pfister SM, Jones DTW, Rippe K, Brors B. 2019. TelomereHunter - in silico estimation of telomere content and composition from Cancer genomes. BMC Bioinformatics 20:272. DOI: https://doi.org/10.1186/s12859-019-2851-0, PMID: 31138115 
Flynn RL, Cox KE, Jeitany M, Wakimoto H, Bryll AR, Ganem NJ, Bersani F, Pineda JR, Suvà ML, Benes CH, Haber DA, Boussin FD, Zou L. 2015. Alternative lengthening of telomeres renders Cancer cells hypersensitive to ATR inhibitors. Science 347:273-277. DOI: https://doi.org/10.1126/science.1257216, PMID: 25593184

Gall JG. 2003. The centennial of the Cajal body. Nature reviews. Molecular cell biology 4:975-980 . DOI: https:// doi.org/10.1038/nrm1262, PMID: 14685175

Ghandi M, Huang FW, Jané-Valbuena J, Kryukov GV, Lo CC, McDonald ER, Barretina J, Gelfand ET, Bielski CM, Li H, Hu K, Andreev-Drakhlin AY, Kim J, Hess JM, Haas BJ, Aguet F, Weir BA, Rothberg MV, Paolella BR, Lawrence MS, et al. 2019. Next-generation characterization of the Cancer cell line encyclopedia. Nature 569: 503-508. DOI: https://doi.org/10.1038/s41586-019-1186-3, PMID: 31068700

Goldman M, Craft B, Hastie M, Repečka K, McDade F, Kamath A, Banerjee A, Luo Y, Rogers D, Brooks AN, Zhu J, Haussler D. 2019. The UCSC xena platform for public and private Cancer genomics data visualization and interpretation. bioRxiv. DOI: https://doi.org/10.1101/326470

Greider CW. 2012. Wnt regulates TERT-putting the horse before the cart. Science 336:1519-1520. DOI: https:// doi.org/10.1126/science.1223785

Greider CW, Blackburn EH. 1985. Identification of a specific telomere terminal transferase activity in Tetrahymena extracts. Cell 43:405-413. DOI: https://doi.org/10.1016/0092-8674(85)90170-9, PMID: 3907856

GTEx Consortium, Demanelis K, Jasmine F, Chen LS, Chernoff M, Tong L, Delgado D, Zhang C, Shinkle J, Sabarinathan M, Lin H, Ramirez E, Oliva M, Kim-Hellmuth S, Stranger BE, Lai TP, Aviv A, Ardlie KG, Aguet F, Ahsan H, Doherty JA, et al. 2020. Determinants of telomere length across human tissues. Science 369: eaaz6876. DOI: https://doi.org/10.1126/science.aaz6876, PMID: 32913074

Hackett JA, Greider CW. 2002. Balancing instability: dual roles for telomerase and telomere dysfunction in tumorigenesis. Oncogene 21:619-626. DOl: https://doi.org/10.1038/sj.onc.1205061, PMID: 11850787

Heaphy CM, de Wilde RF, Jiao Y, Klein AP, Edil BH, Shi C, Bettegowda C, Rodriguez FJ, Eberhart CG, Hebbar S, Offerhaus GJ, McLendon R, Rasheed BA, He Y, Yan H, Bigner DD, Oba-Shinjo SM, Marie SK, Riggins GJ, Kinzler KW, et al. 2011a. Altered telomeres in tumors with ATRX and DAXX mutations. Science 333:425. DOI: https://doi.org/10.1126/science.1207313, PMID: 21719641

Heaphy CM, Subhawong AP, Hong SM, Goggins MG, Montgomery EA, Gabrielson E, Netto GJ, Epstein JI, Lotan TL, Westra WH, Shih I, lacobuzio-Donahue CA, Maitra A, Li QK, Eberhart CG, Taube JM, Rakheja D, Kurman RJ, Wu TC, Roden RB, et al. 2011b. Prevalence of the alternative lengthening of telomeres telomere maintenance mechanism in human Cancer subtypes. The American Journal of Pathology 179:1608-1615. DOI: https://doi.org/10.1016/j.ajpath.2011.06.018, PMID: 21888887

Horn S, Figl A, Rachakonda PS, Fischer C, Sucker A, Gast A, Kadel S, Moll I, Nagore E, Hemminki K, Schadendorf D, Kumar R. 2013. TERT promoter mutations in familial and sporadic melanoma. Science 339:959-961. DOI: https://doi.org/10.1126/science.1230062, PMID: 23348503

Huang FW, Hodis E, Xu MJ, Kryukov GV, Chin L, Garraway LA. 2013. Highly recurrent TERT promoter mutations in human melanoma. Science 339:957-959. DOI: https://doi.org/10.1126/science.1229259, PMID: 23348506

Huang FW, Bielski CM, Rinne ML, Hahn WC, Sellers WR, Stegmeier F, Garraway LA, Kryukov GV. 2015. TERT promoter mutations and monoallelic activation of TERT in Cancer. Oncogenesis 4:e176. DOI: https://doi.org/ 10.1038/oncsis.2015.39, PMID: 26657580

Killela PJ, Reitman ZJ, Jiao Y, Bettegowda C, Agrawal N, Diaz LA, Friedman AH, Friedman H, Gallia GL, Giovanella BC, Grollman AP, He TC, He Y, Hruban RH, Jallo Gl, Mandahl N, Meeker AK, Mertens F, Netto GJ, Rasheed BA, et al. 2013. TERT promoter mutations occur frequently in gliomas and a subset of tumors derived from cells with low rates of self-renewal. PNAS 110:6021-6026. DOI: https://doi.org/10.1073/pnas. 1303607110, PMID: 23530248

Kim NW, Piatyszek MA, Prowse KR, Harley CB, West MD, Ho PL, Coviello GM, Wright WE, Weinrich SL, Shay JW. 1994. Specific association of human telomerase activity with immortal cells and Cancer. Science 266:20112015. DOI: https://doi.org/10.1126/science.7605428, PMID: 7605428

Kim W, Ludlow AT, Min J, Robin JD, Stadler G, Mender I, Lai TP, Zhang N, Wright WE, Shay JW. 2016. Regulation of the human telomerase gene TERT by telomere position Effect-Over long distances (TPE-OLD): Implications for aging and Cancer. PLOS Biology 14:e2000016. DOI: https://doi.org/10.1371/journal.pbio. 2000016, PMID: 27977688

Kim W, Shay JW. 2018. Long-range telomere regulation of gene expression: telomere looping and telomere position effect over long distances (TPE-OLD). Differentiation 99:1-9. DOI: https://doi.org/10.1016/j.diff.2017. 11.005, PMID: 29197683

Lee M, Teber ET, Holmes O, Nones K, Patch AM, Dagg RA, Lau LMS, Lee JH, Napier CE, Arthur JW, Grimmond SM, Hayward NK, Johansson PA, Mann GJ, Scolyer RA, Wilmott JS, Reddel RR, Pearson JV, Waddell N, Pickett HA. 2018. Telomere sequence content can be used to determine ALT activity in tumours. Nucleic Acids Research 46:4903-4918. DOI: https://doi.org/10.1093/nar/gky297, PMID: 29718321

Lee DD, Leão R, Komosa M, Gallo M, Zhang CH, Lipman T, Remke M, Heidari A, Nunes NM, Apolónio JD, Price AJ, De Mello RA, Dias JS, Huntsman D, Hermanns T, Wild PJ, Vanner R, Zadeh G, Karamchandani J, Das S, et al. 2019. DNA hypermethylation within TERT promoter upregulates TERT expression in Cancer. Journal of Clinical Investigation 129:1801. DOI: https://doi.org/10.1172/JCl128527

Listerman I, Sun J, Gazzaniga FS, Lukas JL, Blackburn EH. 2013. The major reverse transcriptase-incompetent splice variant of the human telomerase protein inhibits telomerase activity but protects from apoptosis. Cancer Research 73:2817-2828. DOI: https://doi.org/10.1158/0008-5472.CAN-12-3082, PMID: 23610451 
Lorbeer FK, Hockemeyer D. 2020. TERT promoter mutations and telomeres during tumorigenesis. Current Opinion in Genetics \& Development 60:56-62. DOI: https://doi.org/10.1016/j.gde.2020.02.001, PMID: 32163 830

McClintock B. 1941. The stability of broken ends of chromosomes in Zea mays. Genetics 26:234-282.

DOI: https://doi.org/10.1093/genetics/26.2.234, PMID: 17247004

McClintock B. 1942. The fusion of broken ends of chromosomes following nuclear fusion. PNAS 28:458-463.

DOI: https://doi.org/10.1073/pnas.28.11.458, PMID: 16578057

McDonald ER, de Weck A, Schlabach MR, Billy E, Mavrakis KJ, Hoffman GR, Belur D, Castelletti D, Frias E, Gampa K, Golji J, Kao I, Li L, Megel P, Perkins TA, Ramadan N, Ruddy DA, Silver SJ, Sovath S, Stump M, et al. 2017. Project DRIVE: a compendium of Cancer dependencies and synthetic lethal relationships uncovered by Large-Scale, deep RNAi screening. Cell 170:577-592. DOI: https://doi.org/10.1016/j.cell.2017.07.005, PMID: 2 8753431

Meyers RM, Bryan JG, McFarland JM, Weir BA, Sizemore AE, Xu H, Dharia NV, Montgomery PG, Cowley GS, Pantel S, Goodale A, Lee Y, Ali LD, Jiang G, Lubonja R, Harrington WF, Strickland M, Wu T, Hawes DC, Zhivich VA, et al. 2017. Computational correction of copy number effect improves specificity of CRISPR-Cas9 essentiality screens in Cancer cells. Nature Genetics 49:1779-1784. DOI: https://doi.org/10.1038/ng.3984, PMID: 29083409

Mitchell JR, Cheng J, Collins K. 1999. A box H/ACA small nucleolar RNA-like domain at the human telomerase RNA 3' end. Molecular and Cellular Biology 19:567-576. DOI: https://doi.org/10.1128/MCB.19.1.567, PMID: 9858580

Nagraj VP, Magee NE, Sheffield NC. 2018. LOLAweb: a containerized web server for interactive genomic locus overlap enrichment analysis. Nucleic Acids Research 46:W194-W199. DOI: https://doi.org/10.1093/nar/gky464, PMID: 29878235

Okamoto K, Seimiya H. 2019. Revisiting telomere shortening in Cancer. Cells 8:107. DOI: https://doi.org/10. 3390/cells8020107

Olovnikov AM. 1973. A theory of marginotomy the incomplete copying of template margin in enzymic synthesis of polynucleotides and biological significance of the phenomenon. Journal of Theoretical Biology 41:181-190. DOI: https://doi.org/10.1016/0022-5193(73)90198-7, PMID: 4754905

Pan J, Meyers RM, Michel BC, Mashtalir N, Sizemore AE, Wells JN, Cassel SH, Vazquez F, Weir BA, Hahn WC, Marsh JA, Tsherniak A, Kadoch C. 2018. Interrogation of mammalian protein complex structure, function, and membership using Genome-Scale fitness screens. Cell Systems 6:555-568. DOI: https://doi.org/10.1016/j.cels. 2018.04.011, PMID: 29778836

PCAWG-Structural Variation Working Group, PCAWG Consortium, Sieverling L, Hong C, Koser SD, Ginsbach P, Kleinheinz K, Hutter B, Braun DM, Cortés-Ciriano I, Xi R, Kabbe R, Park PJ, Eils R, Schlesner M, Brors B, Rippe K, Jones DTW, Feuerbach L. 2020. Genomic footprints of activated telomere maintenance mechanisms in Cancer. Nature Communications 11:733. DOI: https://doi.org/10.1038/s41467-019-13824-9, PMID: 32024 817

Pereboeva L, Hubbard M, Goldman FD, Westin ER. 2016. Robust DNA damage response and elevated reactive oxygen species in TINF2-Mutated dyskeratosis congenita cells. PLOS ONE 11:e0148793. DOI: https://doi.org/ 10.1371/journal.pone.0148793, PMID: 26859482

Ramamoorthy M, Smith S. 2015. Loss of ATRX suppresses resolution of telomere cohesion to control recombination in ALT Cancer cells. Cancer Cell 28:357-369. DOI: https://doi.org/10.1016/j.ccell.2015.08.003, PMID: 26373281

Rosenfeld JA, Wang Z, Schones DE, Zhao K, DeSalle R, Zhang MQ. 2009. Determination of enriched histone modifications in non-genic portions of the human genome. BMC Genomics 10:143. DOI: https://doi.org/10. 1186/1471-2164-10-143, PMID: 19335899

Rowland TJ, Dumbović G, Hass EP, Rinn JL, Cech TR. 2019. Single-cell imaging reveals unexpected heterogeneity of telomerase reverse transcriptase expression across human Cancer cell lines. PNAS 116 18488-18497. DOI: https://doi.org/10.1073/pnas.1908275116, PMID: 31451652

Rowland TJ, Bonham AJ, Cech TR. 2020. Allele-specific proximal promoter hypomethylation of the telomerase reverse transcriptase gene (TERT) associates with TERT expression in multiple cancers. Molecular Oncology 14: 2358-2374. DOI: https://doi.org/10.1002/1878-0261.12786, PMID: 33245585

Ruthenburg AJ, Li H, Patel DJ, Allis CD. 2007. Multivalent engagement of chromatin modifications by linked binding modules. Nature Reviews Molecular Cell Biology 8:983-994. DOI: https://doi.org/10.1038/nrm2298, PMID: 18037899

Salgado C, Roelse C, Nell R, Gruis N, Doorn R, Velden P. 2019. Interplay between TERT promoter mutations and methylation culminates in chromatin accessibility and TERT expression. bioRxiv. DOI: https://doi.org/10.1101/ 859892

Sheffield NC, Bock C. 2016. LOLA: enrichment analysis for genomic region sets and regulatory elements in R and bioconductor. Bioinformatics 32:587-589. DOI: https://doi.org/10.1093/bioinformatics/btv612, PMID: 2650 8757

Song Q, Decato B, Hong EE, Zhou M, Fang F, Qu J, Garvin T, Kessler M, Zhou J, Smith AD. 2013. A reference methylome database and analysis pipeline to facilitate integrative and comparative epigenomics. PLOS ONE 8: e81148. DOI: https://doi.org/10.1371/journal.pone.0081148

Stern JL, Theodorescu D, Vogelstein B, Papadopoulos N, Cech TR. 2015. Mutation of the TERT promoter, switch to active chromatin, and monoallelic TERT expression in multiple cancers. Genes \& Development 29:22192224. DOI: https://doi.org/10.1101/gad.269498.115, PMID: 26515115 
Stern JL, Paucek RD, Huang FW, Ghandi M, Nwumeh R, Costello JC, Cech TR. 2017. Allele-Specific DNA methylation and its interplay with repressive histone marks at Promoter-Mutant TERT genes. Cell Reports 21: 3700-3707. DOI: https://doi.org/10.1016/j.celrep.2017.12.001, PMID: 29281820

Takakura M, Kyo S, Kanaya T, Tanaka M, Inoue M. 1998. Expression of human telomerase subunits and correlation with telomerase activity in cervical Cancer. Cancer Research 58:1558-1561. PMID: 9537264

Tao Y, Kang B, Petkovich DA, Bhandari YR, In J, Stein-O'Brien G, Kong X, Xie W, Zachos N, Maegawa S, Vaidya H, Brown S, Chiu Yen RW, Shao X, Thakor J, Lu Z, Cai Y, Zhang Y, Mallona I, Peinado MA, et al. 2019. Aginglike spontaneous epigenetic silencing facilitates wnt activation, stemness, and Braf ${ }^{\mathrm{6}}{ }^{00 \mathrm{E}}$-Induced Tumorigenesis. Cancer Cell 35:315-328. DOI: https://doi.org/10.1016/j.ccell.2019.01.005, PMID: 30753828

Tate JG, Bamford S, Jubb HC, Sondka Z, Beare DM, Bindal N, Boutselakis H, Cole CG, Creatore C, Dawson E, Fish P, Harsha B, Hathaway C, Jupe SC, Kok CY, Noble K, Ponting L, Ramshaw CC, Rye CE, Speedy HE, et al. 2019. COSMIC: the catalogue of somatic mutations in Cancer. Nucleic Acids Research 47:D941-D947. DOI: https://doi.org/10.1093/nar/gky1015, PMID: 30371878

Tsherniak A, Vazquez F, Montgomery PG, Weir BA, Kryukov G, Cowley GS, Gill S, Harrington WF, Pantel S, KrillBurger JM, Meyers RM, Ali L, Goodale A, Lee Y, Jiang G, Hsiao J, Gerath WFJ, Howell S, Merkel E, Ghandi M, et al. 2017. Defining a Cancer dependency map. Cell 170:564-576. DOI: https://doi.org/10.1016/j.cell.2017.06. 010, PMID: 28753430

UK10K Consortium, Ding Z, Mangino M, Aviv A, Spector T, Durbin R. 2014. Estimating telomere length from whole genome sequence data. Nucleic Acids Research 42:e75. DOI: https://doi.org/10.1093/nar/gku181, PMID: 24609383

Van der Auwera GA, Carneiro MO, Hartl C, Poplin R, Del Angel G, Levy-Moonshine A, Jordan T, Shakir K, Roazen D, Thibault J, Banks E, Garimella KV, Altshuler D, Gabriel S, DePristo MA. 2013. From fastQ data to high-confidence variant calls: the genome analysis toolkit best practices pipeline. Current Protocols in Bioinformatics 43:43. DOI: https://doi.org/10.1002/0471250953.bi1110s43, PMID: 25431634

Venteicher AS, Abreu EB, Meng Z, McCann KE, Terns RM, Veenstra TD, Terns MP, Artandi SE. 2009. A human telomerase holoenzyme protein required for cajal body localization and telomere synthesis. Science 323:644648. DOI: https://doi.org/10.1126/science.1165357, PMID: 19179534

Verdun RE, Karlseder J. 2007. Replication and protection of telomeres. Nature 447:924-931. DOI: https://doi. org/10.1038/nature05976, PMID: 17581575

Wan M, Qin J, Songyang Z, Liu D. 2009. OB fold-containing protein 1 (OBFC1), a human homolog of yeast Stn1, associates with TPP1 and is implicated in telomere length regulation. Journal of Biological Chemistry 284: 26725-26731. DOl: https://doi.org/10.1074/jbc.M109.021105, PMID: 19648609

Wu KJ, Grandori C, Amacker M, Simon-Vermot N, Polack A, Lingner J, Dalla-Favera R. 1999. Direct activation of TERT transcription by c-MYC. Nature genetics 21:220-224 . DOl: https://doi.org/10.1038/6010, PMID: 9988278

Yang W, Soares J, Greninger P, Edelman EJ, Lightfoot H, Forbes S, Bindal N, Beare D, Smith JA, Thompson IR, Ramaswamy S, Futreal PA, Haber DA, Stratton MR, Benes C, McDermott U, Garnett MJ. 2013. Genomics of drug sensitivity in Cancer (GDSC): a resource for therapeutic biomarker discovery in Cancer cells. Nucleic Acids Research 41:D955-D961. DOI: https://doi.org/10.1093/nar/gks1111

Yu GL, Bradley JD, Attardi LD, Blackburn EH. 1990. In vivo alteration of telomere sequences and senescence caused by mutated Tetrahymena telomerase RNAs. Nature 344:126-132. DOI: https://doi.org/10.1038/ 344126a0, PMID: 1689810

Yuan X, Larsson C, Xu D. 2019. Mechanisms underlying the activation of TERT transcription and telomerase activity in human cancer: old actors and new players. Oncogene 38:6172-6183. DOl: https://doi.org/10.1038/ s41388-019-0872-9, PMID: 31285550

Zhang A, Zheng C, Lindvall C, Hou M, Ekedahl J, Lewensohn R, Yan Z, Yang X, Henriksson M, Blennow E, Nordenskjöld M, Zetterberg A, Björkholm M, Gruber A, Xu D. 2000. Frequent amplification of the telomerase reverse transcriptase gene in human tumors. Cancer Research 60:6230-6235. PMID: 11103775

Zhu Y, Tomlinson RL, Lukowiak AA, Terns RM, Terns MP. 2004. Telomerase RNA accumulates in cajal bodies in human Cancer cells. Molecular Biology of the Cell 15:81-90. DOl: https://doi.org/10.1091/mbc.e03-07-0525, PMID: 14528011 